\title{
Identification of Hypoxia-Regulated Genes in the Liver of Common Sole (Solea solea) Fed Different Dietary Lipid Contents
}

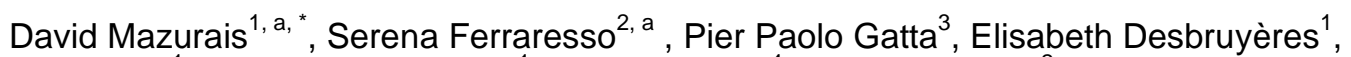 \\ Armelle Severe ${ }^{1}$, Charlotte Corporeau ${ }^{1}$, Guy Claireaux ${ }^{4}$, Luca Bargelloni ${ }^{2}$, Jose-Luis Zambonino- \\ Infante $^{1}$
}

1 Ifremer, UMR 6539 LEMAR, Unité de Physiologie Fonctionnelle des Organismes Marins, Ifremer, CS 10070,
29280 , Plouzané, France
2 Department of Comparative Biomedicine and Food Science, University of Padova, Viale dell'Università 16,
35020 , Legnaro, Italy
3 Dipartimento di Scienze Mediche Veterinarie, Alma Mater Studiorum Università di Bologna, Via Tolara di Sopra,
50,40064 , Ozzano Emilia, Bologna, Italy
4 LEMAR UMR 6539, Institut Universitaire Européen de la Mer, Université de Bretagne Occidentale, Technopôle
Brest Iroise, Place Nicolas Copernic, Plouzané, 29280, France

*: Corresponding author : David Mazurais, email address : david.mazurais@ifremer.fr

${ }^{a}$ David Mazurais and Serena Ferraresso contributed equally to this paper.

\begin{abstract}
:
Coastal systems could be affected by hypoxic events brought about by global change. These areas are essential nursery habitats for several fish species including the common sole (Solea solea L.). Tolerance of fish to hypoxia depends on species and also on their physiological condition and nutritional status. Indeed, high dietary lipid content has been recently shown to negatively impact the resistance of sole to a severe hypoxic challenge. In order to study the molecular mechanisms involved in the early response to hypoxic stress, the present work examined the hepatic transcriptome in common sole fed diets with low and high lipid content, exposed to severe hypoxia. The activity of AMP-activated protein kinase (AMPK) was also investigated through the quantification of threonine172 phosphorylation in the alpha subunit. The results show that hypoxia consistently regulates several actors involved in energy metabolism pathways and particularly AMPKa, as well as some involved in cell growth and maintenance or unfolded protein response. Our findings reveal that (1) the expression of genes involved in biological processes with high energy cost or implicated in aerobic ATP synthesis was down-regulated by hypoxia, contrary to genes involved in neoglucogenesis or in angiogenesis, (2) the consumption of high lipid induced regulation of metabolic pathways going against this energy saving, and (3) this control was fine-tuned by the regulation of several transcriptomic factors. These results provide insight into the biological processes involved in the hepatic response to hypoxic stress and underline the negative impact of high lipid consumption on the tolerance of common sole to hypoxia.
\end{abstract}

Keywords: Fish ; Hypoxia ; Nutrition ; Liver ; Transcriptome ; AMPK 
Over the past 50 years, the intensification of anthropogenic activities along riversides and coastlines, combined with global warming, has been connected with the increasing duration and severity of environmental hypoxia in many coastal marine ecosystems. Consequently, hypoxia is now considered as one of the most pressing environmental issues worldwide $\mathrm{Wu}$ 2002). Shallow coastal ecosystems are nursery areas for many benthic fish species and it is believed that hypoxic events can potentially impact them.

A large number of studies have been conducted to examine the physiological consequences and regulatory mechanisms that respond following a reduction in oxygen availability (reviewed by Richards et al. 2009). Although the physiological response to hypoxia varies among fish species (Mandic et al. 2009), it is accepted that they follow a general strategy aimed at inhibiting oxygen- and ATP-demanding metabolic pathways, while favouring the activation of oxygen-independent ATP-production pathways (reviewed by Almeida-Val et al. 2006; Bickler and Buck 2007; López-Barneo et al. 2010; Richards 2011). All these studies showed the common feature that this transition requires adequate stores of glucose, generally in the form glycogen, such as that found in the liver (Richards 2011).

The liver plays a central role in synthesizing or converting molecules that are utilized elsewhere to maintain homeostasis, and in regulating energy balance. Since the regulations of enzyme activity required for metabolic adaptation to hypoxia have been shown to be related to the differential transcription of mRNA (Semenza et al. 1996), a large scale investigation of gene expression would improve understanding of the overall impact of hypoxia on fish physiology. Previous studies performed on hepatic tissue have revealed impacts of hypoxia exposure on the expression of genes involved in energy metabolism (i.e., glucose metabolism), cell growth and proliferation, protein degradation and oxygenase activities (Gracey et al. 2001; Ju et al. 2007; Leveelahti et al. 2011; Everett et al. 2012). As in mammals, Hypoxia Inducible Factors (HIFs) are thought to be involved as transcription factors in the coordination of molecular responses in fish (see review by Nikinmaa and Rees 2005), especially in the liver (Gracey et al. 2001). AMPK, a nutrient and energy sensor that maintains energy homeostasis, is also suggested to play a central role in coordinating the metabolic responses of fish exposed to severe hypoxia (Jibb and Richards 2008). However, numerous key actors and cellular transduction pathways involved in the transcriptional control of metabolism during hypoxia are still unknown.

In the present study, we applied microarray-based gene expression profiling to investigate the short term transcriptional response of metabolic reprogramming during an acute, severe hypoxia challenge in juvenile common sole (Solea solea). Moreover, phosphorylation of threonine 172 in the alpha subunit, which is a key determinant of AMPK activity (Hardie 2008), was assessed on the same fish. Common sole is a flatfish of the Soleidae family, which is particularly exposed to hypoxia events during juvenile stages because estuaries have been identified as essential nurseries for this species (Le Pape et al. 2003). Common sole can tolerate quite severe drops in ambient oxygenation and does not show a strong avoidance response to hypoxic estuarine environments (Cannas et al. 2007). Previous studies aiming to better characterise the physiological response of common sole to hypoxia revealed a typical metabolic depression (Dalla Via et al. 1994; 1997). Moreover, recent studies have suggested that the dietary lipid content as a factor influencing hypoxia tolerance of common sole (McKenzie et al. 2008; Zambonino-Infante et al. 2013). In particular, Zambonino-Infante et al. (2013) showed that juvenile sole exhibited a lower tolerance to hypoxia when fed a lipid-rich diet. Even though this effect can probably be related to the known low nutritional tolerance of sole species to high lipid ingestion, which induces perturbation of their energy metabolism, the molecular actors and biological processes 
involved in these regulations are poorly documented. To improve our understanding of these processes, we investigated the hepatic transcriptome in juvenile sole fed diets with low and high lipid contents.

\section{Material and methods}

\subsection{Feeding trial, hypoxia challenge stress and sampling}

Experiments were conducted in strict compliance with the Guide for the Care and Use of Laboratory Animals (National Research Council 2010). One thousand North Sea sole (Solea solea) eggs were purchased from a commercial hatchery (SOLEA BV, ljmuiden, Netherlands) and brought to the Ifremer larval rearing facility in Brest. Details on larval rearing equipment and conditions can be found in Zambonino-Infante et al. (2013). Eight months post hatching, 160 individuals were randomly drawn from the rearing tanks, tagged subcutaneously (passive integrated transponder: "PIT-tag") and distributed among eight 67-L tanks. For two months, these fish were maintained at $16^{\circ} \mathrm{C}$ and fed with commercial diets containing either $11 \%$ lipids (BP Finition Label Extrudé, Le Gouessant, France) (I-group) or $20 \%$ lipids (Neo Grower Extra Marin, Le Gouessant, France) (L-group) with four tanks per dietary group.

Prior to the hypoxia challenge test, 74 individuals selected at random (37 from each dietary group) were moved into a single tank $\left(1 \mathrm{~m}^{3}\right)$ and left undisturbed and unfed for $48 \mathrm{~h}$. The blood and liver of 36 of these individuals (18 from each dietary group) were sampled in normoxic conditions for biochemical and molecular analysis, respectively.

The hypoxia challenge was applied to the remaining 38 fishes (19 from each dietary group) and consisted in decreasing water oxygenation from $100 \%$ air saturation to $10 \%$ air saturation within one hour, followed by a slower descent to $1 \%$ air sat. over the last 30 minutes. Ambient oxygenation was controlled by bubbling nitrogen at the intake of a submersible pump placed in the tank. After 90 minutes of hypoxia, fish were removed, identified (PIT-tag reading) and their blood and liver sampled. The resulting four final experimental groups were named as follows: Normoxia l-group ( $\mathrm{NI})$, Normoxia L-group (NL), Hypoxia l-group $(\mathrm{HI})$, Hypoxia L-group $(\mathrm{HL})$. The experimental design was therefore a 2-way full factorial design between two juvenile dietary lipid contents and two oxygenation conditions. Data related to blood (biochemical) parameters measured in these groups were recently published (Zambonino-Infante et al. 2013).

\subsection{RNA extraction and purification}

Liver tissue from 74 individuals was homogenised in $2 \mathrm{ml}$ Extract All Reagent (Eurobio, Courtaboeuf, France). Total RNA was isolated following the manufacturer's instructions and quantified by measuring absorbance at $260 \mathrm{~nm}$ in a spectrophotometer (NanoDrop, Labtech, France). RNA integrity was also evaluated using a Bioanalyzer 2100 (Agilent, Santa Clara, USA) analysis. Following the evaluation of RNA integrity, 71 samples (with RIN> 8) were kept for microarray hybridization: 18 from group NI, 18 from group NL, 16 from group $\mathrm{HI}$ and 19 from group $\mathrm{HL}$.

\subsection{Quantification of Threonine 172 phosphorylation of AMPKa}

Total protein extracts were obtained according to Corporeau and Auffret (2003) from the same 74 liver samples. Briefly, to solubilize proteins, powdered tissues were homogenized in 
a lysis buffer containing phosphatase and protease inhibitors $(150 \mathrm{mM} \mathrm{NaCl}, 10 \mathrm{mM}$ Tris, $\mathrm{pH}$ 7.4, $1 \mathrm{mM}$ EDTA, $1 \mathrm{mM}$ EGTA, 1\% Triton X-100, 0.5\% Igepal, $5 \mathrm{ml}$ phosphatase inhibitor cocktail II, 2 tablets of cOmplete EDTA-free protease inhibitor cocktail; pH 8.8 at $4^{\circ} \mathrm{C}$ ). Total protein extracts were then quantified using a DC protein assay (Biorad), and protein concentrations were determined quantitatively using 96-well micro-plates (Nunc) and a micro-plate reader connected to KC4 v3 software (Bio-Tek Instruments, Inc).

Prior to quantification of Threonine 172 phosphorylation of AMPK $\alpha$, each protein lysate was adjusted to a final concentration of $3.5 \mathrm{mg} / \mathrm{ml}$ by adding lysis buffer. Thr 172 phosphorylation of AMPK $\alpha$ was detected by the PathScan ${ }^{\circledR}$ Phospho-AMPK $\alpha$ (Thr172) Sandwich ELISA Kit following manufacturer's instructions (Cell Signaling Technology, ref \#7959). Western blot analysis from protein lysates of sole tissues was previously performed using Rat Anti-AMPKa (23A3) (Rabbit mAb, \#2603, Cell Signaling) to ensure that the ELISA assay reported correctly threonine 172 phosphorylation of AMPK in sole. A specific band was detected at approximately $60 \mathrm{kDa}$ confirming high AMPKa amino-acid sequence identity $(>90 \%)$ between sole and rat species (Additional file 1).

\subsection{Microarray design}

Gene expression profiling of $S$. solea samples was conducted using an updated version of the Agilent-036353 S. solea DNA microarray (GPL16124).

Microarray design was carried out basis of 25,252 contigs (isotigs) and 66,026 singletons (with a minimal length of $200 \mathrm{nt}$ ) obtained from the Roche 454 sequencing of a normalized cDNA library of sole larval stages (from 1 to 33 days post hatching) and adult tissues (Ferraresso et al., submitted data). All assembled isotigs are stored in the public database Transcriptome Shotgun Assembly Sequence Database (TSA, http://www.ncbi.nlm.nih.gov/genbank/tsa) under accession number GAAQ00000000 (submitted), while singletons can be directly retrieved from the NCBI Sequence Read Archive (SRA) under accession number SRA058691.

Transcript annotation for both isotigs and singletons was implemented through blastx searches (cut off e-value of < $1.0 \mathrm{E}-5$ ) against high quality draft proteomes of Danio rerio, Gasterosteus aculeatus, Oryzias latipes, Takifugu rubripes, Tetraodon nigroviridis and Homo sapiens, available on the Ensembl Genome Browser (release 56), and against the SWISSPROT database. A schematic representation of the approach used for microarray design is given in Additional File 2. Briefly, all annotated isotigs were screened for redundancy using Ensembl Protein IDs. Two or more transcripts were considered "redundant" when they showed the same annotation as at least 3 fish species out of 5 , by considering Ensembl Protein ID of five fish species ( $D$. rerio, G. aculeatus, $O$. latipes, $T$. nigroviridis, $T$. rubripes) and only the longest was considered for microarray design. The same approach was followed for filtering out redundant singletons. A total of 8,750 isotigs and 5,800 singletons were finally represented in the array.

A total of 14,701 probes, targeting 14,531 transcripts, were successfully designed; 8,918 of these had been previously employed on the first version of the array (GEO acc: GPL16124). Probe sequences and further details on the microarray platform can be found in the GEO repository (http://www.ncbi.nlm.nih.gov/geo/) under accession number GPL16714.

\subsection{Microarray hybridization and data analysis}

Sample labeling and hybridization were performed according to the Agilent One-Color Microarray-Based Gene Expression Analysis protocol for $8 \times 15 \mathrm{~K}$ slide format. 
Briefly, for each sample 100 ng total RNA were linearly amplified and labeled with Cy3dCTP. A mixture of 10 different viral poly-adenylated RNAs (Agilent Spike-In Mix) was added to each RNA sample before amplification and labeling, to monitor microarray analysis workflow. Labeled cRNA was purified with a Qiagen RNeasy Mini Kit, and sample concentration

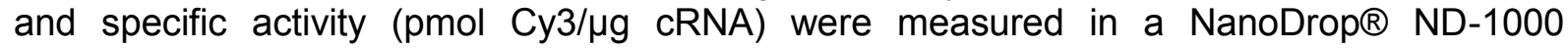
spectrophotometer. A total of $600 \mathrm{ng}$ labeled cRNA were prepared for fragmentation by adding $5 \mu \mathrm{l} 10 \mathrm{X}$ blocking agent and $1 \mu \mathrm{l} 25 \mathrm{X}$ fragmentation buffer, heated to $60^{\circ} \mathrm{C}$ for $30 \mathrm{~min}$, and finally diluted by addition of $25 \mu \mathrm{l} 2 \mathrm{X}$ GE Hybridization buffer. A volume of $40 \mu \mathrm{l}$ hybridization solution was then dispensed in the gasket slide and assembled with the microarray slide (each slide containing eight arrays). Slides were incubated for $17 \mathrm{~h}$ at $65^{\circ} \mathrm{C}$ in an Agilent hybridization oven, then removed from the hybridization chamber, quickly submerged in GE Wash Buffer 1 for disassembly of the slides and then washed in GE wash buffer 1 for approximately 1 minute followed by one additional wash in pre-warmed $\left(37^{\circ} \mathrm{C}\right)$ GE wash buffer 2 .

Hybridized slides were scanned at $5 \mu \mathrm{m}$ resolution using an Agilent G2565BA DNA microarray scanner. Default settings were modified to scan each slide twice at two different sensitivity levels (XDR Hi 100\% and XDR Lo 10\%). The two linked images generated were analyzed together and the data were extracted and background subtracted using the standard procedures in the Agilent Feature Extraction (FE) Software version 9.5.1. Cyclic loess normalization was performed using $R$ statistical software. Spike-in control intensities were used to monitor the normalization procedure.

Raw and normalized fluorescence data of all microarray experiments were deposited in the GEO database under accession numbers GSE44579.

\subsection{Statistical analysis}

Two-way ANOVA (stat soft) was used to compare the phosphorylation levels of AMPK $\alpha$ between groups. The microarray data were also analysed by two-way ANOVA using Tmev (TIGR MultiExperiment Viewer) statistical software, and gene expression was considered significantly different when $p$-value $<0.005$. No multiple test correction (i.e. Bonferroni) was employed as previous analyses indicated that such corrections could be over-conservative (Leaver et al., 2008). However, in order to minimize the type 1 error and related false discovery rates (FDR), $p$-value was fixed at 0.005 . Significant enrichment of GO biological process categories were tested for using EASE software (version 2.0). Benjamini correction was applied for statistical analysis related to GO enrichment.

\section{Results and discussion}

\subsection{Impact of hypoxic challenge}

The main objective of the present study was to investigate the short-term response of the liver transcriptome to an acute hypoxic challenge in common sole fed diets of different lipid content. The reliability of datasets was confirmed by the reproducibility of signal obtained from redundant transcript (data not shown). By examining genes regulated by the "hypoxia" factor, we revealed the molecular actors and related processes underlying the physiological response of common sole to hypoxia in liver tissue. ANOVA revealed that 1202 genes (FDR $<7 \%$, with a p-value of 0.005 ) were significantly regulated following hypoxia, amounting to $8 \%$ of the total spotted genes (table 1 and figure 1). Among these regulated genes, 514 were up-expressed under hypoxic conditions and 688 were down-expressed (additional file 3). Gene Ontology enrichment analysis, which enables the identification of GO terms 
significantly enriched in the input entity list when compared to the whole array dataset, was performed providing evidence for which biological processes may be particularly altered by hypoxia factor. Four significant GO terms, all interrelated, were identified: carbohydrate metabolism, glucose metabolism, energy pathways and molecular process related to oxidoreductase activity (table 2). Based on the GO categorisation, part of the up-regulated genes were related to gluconeogenesis pathway, regulation of cell growth and maintenance, sulfur amino acid biosynthesis, blood vessel development or transcription regulator activity; among down-regulated genes these were mitochondrial ATP synthesis, TCA (tricarboxylic acid cycle) intermediate metabolism, glycolysis, lipid biosynthesis, and protein folding (tables 3 ). The present results revealed an important gene-based metabolic reprogramming characterized by the down-regulation of energy-demand and aerobic energy-supply pathways associated with an induction of processes involved in the supply of anaerobic metabolic substrates and cell rescue.

\subsubsection{Inhibition of genes related to aerobic ATP supply and energy consumption}

We found numerous genes involved in energy metabolism down-regulated by hypoxia (table 3 ). These include four genes involved in ATP synthesis-coupled electron transport (NADH dehydrogenase ubiquinones: five NDUF genes) and four genes involved in the TCA cycle (ACLY, GAD1, IDH1 and ME1). These down-regulations are consistent with the known reduction of energy production by aerobic metabolic pathways in fish subjected to hypoxic conditions (Krumschnabel et al. 2000). Such down-regulations are also well documented in fish (Wright et al. 1989; Martinez et al. 2006), including common sole (Dalla Via et al. 1994; Van den Thillart et al. 1994), and inhibition of aerobic pathways has been seen to be concomitant with an activation of anaerobic ATP-generating pathways which use glucose as the main substrate. In agreement with these previous results, we found that several genes up-regulated by hypoxia were involved in the key steps of the gluconeogenesis pathway (PCK1 and G6PC) and in the metabolism of glucogenic amino acid, such as the transaminases (AADAT, GOT1, OAT, HAL and TAT) (table 3). Our transcriptomic data, which suggest the stimulation of the expression of genes implicated in glucose synthesis through gluconeogenesis, are consistent with the significant hypoxia-induced increase of glycemia that we measured in these fish (Zambonino-Infante et al., 2013). They are also in agreement with previous transcriptomic results on the liver of longjaw mudsucker (Gracey et al. 2001). However, contrary to data obtained by Gracey and collaborators revealing a stimulation of genes involved in hepatic glycolysis, we observed a significant down-regulation of several genes (ENO1, G6PD, HK2, LDHA, LDHB, PFKL, PGD, PGK1, PKM2 and TALDO1) involved in glucose catabolism pathways, including glycolysis (table 3 ). The inhibition of glucose catabolism in the liver points to a strategy of re-localization of energy supply in sole. This strategy could consist in maintaining high blood glucose levels for anaerobic ATP production in priority organs, such as the brain and heart, when survival is at stake.

Interestingly, we also found under hypoxic conditions higher hepatic levels of threonine 172 phosphorylation of AMP-activated protein kinase (AMPK $\alpha$ ), which is an energy sensor protein kinase that plays a key role in maintaining cellular energy balance (figure 2). Considering that this phosophorylation is a key determinant of AMPK activity (Hardie 2008), our data are consistent with the stimulation of AMPK activity observed by Jibb and Richards (2008) in severely hypoxic goldfish. It is well documented that AMPK inhibits energetically expensive anabolic processes, such as protein, glycogen or fatty acid synthesis and cell growth and proliferation, in response to reduction of intracellular ATP levels (Mihaylova and Shaw 2011). In agreement with the present AMPK $\alpha$ stimulation, we observed 19 genes involved in lipid biosynthesis down-regulated and several genes implicated in the suppression of cell growth and proliferation, such as IGFBP-1, GRB10, DUSP1 and DUSP6, TOB1 and BTG-1 (table 3), up-regulated under hypoxic conditions. While inductions of 
IGFBP-1 and GRB10, have been shown to inhibit in vivo IGF action, particularly under hypoxic conditions (Tazuke et al. 1998; Liu and Roth 1995; Kajimura et al. 2005), mitogenactivated proteins DUSP1 and DUSP 6 are known to inactivate the ERK group of MAP kinase involved in cell growth stimulation. The stimulation of IGFBP-1 expression that limits IGF-dependent decrease of growth hormone receptor (GHR) expression (Min et al. 1996) can be related to the increase in hepatic GHR mRNA level that we observed during hypoxia (table 3). TOB1 and BGT-1 are also known to suppress growth through their anti-proliferative function (Ho et al. 2010; Kamaid and Giráldez 2008). The impact of hypoxia on the expression of genes involved in cell growth and proliferation supports the view that fish experiencing reduced oxygen availability must reallocate energy from growth toward lifesustaining processes.

\subsubsection{Induction of vascularization}

Among the regulated genes, we found some actors involved in the regulation of vascular endothelial cells (table 3). GRB10, known to negatively regulate the insulin pathway, is also involved in angiogenesis by regulating the KDR/VEGFR-2 signalling pathway. The upregulation of this gene suggests a stimulation of vascular endothelial cell development. In line with this view, we also noted the stimulation of several actors involved in blood vessel development including ERBB4 and EGFR genes, both of which play an essential role as a cell surface receptor of EGF (Russell et al. 1999). ERBB have been shown to play a key role in the regulation of angiogenesis by inducing the vascular endothelial growth factor (VEGF) (Yen et al. 2002). Even though we did not observe any significant differential expression of VEGF in the present work, we found a positive regulation by hypoxia challenge of PRKCA, PDGFRB, FOXF1, JAG1, RAMP2 and STAB1 (table 3), all known to be involved in blood vessel development (Adachi and Tsujimoto 2002; Ichikawa-Shindo et al. 2008; Stankiewicz et al. 2009; Wang et al. 2002; Zimrin et al. 1996). This stimulation of blood vessel formation during hypoxia, which has been widely documented in vertebrates including fish species (reviewed by Nikinmaa and Rees 2005), reflects the need to optimize oxygen supply to tissues. However, contrary to what has been shown in previous studies performed in rainbow trout by Marinsky et al. (1990) or in the euryoxic fish Gillichthys mirabilis by Gracey et al. (2001), we could not find any significant regulation of actors involved in iron or oxygen binding. It is likely that these regulations, even if they exist in the liver of common sole, do not represent the first line of adaptive strategy at the transcriptomic level.

As they highlight the impact of a hypoxic stress on several processes involved in the regulation of cellular metabolism and growth, as well as oxygen delivery to tissue cells, these data are consistent with previous reports performed on the liver of different fish species (Gillichthys mirabilis: Gracey et al. 2001; Oryzias latipes: Ju et al. 2007; Gasterosteus aculeatus: Leveelahti et al. 2011; Fundulus grandis: Everett et al. 2012).

\subsubsection{Regulation of redox potential}

It is noteworthy that some of the hypoxia-stimulated genes are also involved in sulfur amino acid biosynthesis (CBS and CTH) and in the glutathione metabolism process (GGT1) (table 3 ). This regulation probably allows an optimal intracellular glutathione level to be maintained, contributing to cellular antioxidant defense mechanism under hypoxic conditions. In agreement with the above, our transcriptomic data also revealed the differential expression of 56 genes with oxidoreductase activity (table 2), reinforcing the link between reduced oxygen availability and the regulation of redox potential. Altogether, such transcriptomic features can be related to the increase in mitochondrial reactive oxygen species (ROS) production classically observed in hypoxia (Chandel et al. 1998, Mansfield et al. 2005). 


\subsubsection{Large scale regulation of the hepatic transcriptome by transcriptomic factors}

As mentioned in table 1 , our results indicate that a large number of genes $(n=1202)$ are significantly regulated 90 minutes after the onset of hypoxia. The immediate large-scale effect of hypoxia on the hepatic transcriptome may be partly explained by early regulation of the expression of several transcription factors (i.e., JUN, FOS, EGR1, HIF3, CREB, ATF7IP and CEBPD) listed in table 3 . These transcription factors are indeed involved in a cascade of regulation involving a battery of genes that act in concert to facilitate the supply of oxygen and nutrients, regulate energy metabolism and promote cell survival and growth control (Hochachka et al. 1996; Cummins and Taylor 2005). This result demonstrates that, in addition to the regulations occurring at the post-transcriptomic level (van den Beucken et al. 2011), a severe hypoxic stress has a rapid and profound effect on the hepatic transcriptome.

\subsubsection{Activation of the unfolded protein response (UPR)}

We found several genes involved in protein folding (9 genes) or related to endoplasmic reticulum (RE) (21 genes) down-regulated by hypoxia challenge (table 3 ). In vertebrates it is well documented that regulation of the protein folding process is associated with RE stress triggers UPR (Kim et al. 2008). Interestingly, HSPA5 was shown to be up-regulated following hypoxia (table 3). HSPA5 is a key calcium-dependant chaperone involved in the setting off of the downstream signalling of the UPR through its association with IRE1, ATF6 and PERK (Kim et al. 2008). In a similar way as in the results obtained by Tagliavacca et al. (2012) and Kim et al. (2008) in mammals, the present data suggest that these pathways were also triggered by hypoxia in fish. In mammals, it is well documented that the PERK pathway is initiated by the phosphorylation of elF $2 \alpha$, thereby blocking protein translation and activating the transcription factor ATF4. ATF4 controls the expression of genes involved in redox balance, amino acid metabolism, protein folding and apoptosis (Ameri and Harris 2008). In the present work, we observed up-regulation of challenge target genes of ATF4 such as the previously mentioned folding protein HSPA5, the amino acid transporter SLC7A3 as well as IGFBP-1 and GRB10, which are implicated in cell growth mechanisms (Luo et al. 2003; Harding et al. 2003) (table 3). This suggests that the PERK pathway is also activated during hypoxia in sole. With respect to the other UPR pathways, it is known that ATF6 and XBP1 in mammals are able to transactivate genes encoding chaperones and proteins involved in endoplasmic reticulum-associated degradation (ERAD) (Jäger et al. 2012; Kim et al. 2008). The stimulation of the ATF6-target gene Herpud1 (table 3), involved in ERAD (Ma and Hendershot 2004), also suggests the activation of the ATF6 pathway within the 90 minutes of hypoxia tested in the present study.

While UPR consists of mechanisms decreasing the synthesis of proteins and their influx into the ER, we found only a few genes involved in this process that were regulated during environmental hypoxia. This observation suggests that the main mechanisms responsible for the decrease in protein synthesis shortly after the initiation of hypoxia require posttranscriptional regulation.

Our data revealed that most of the molecular actors involved in ER stress, as they were described for mammals, can also be found in common sole. Moreover, these data are totally consistent with a recent study of Ishikawa et al. (2011) indicating that the process of UPR is conserved across vertebrates including fish. 


\subsection{Impact of dietary lipid content}

The second objective of this work was to determine the biological processes underlying the lower tolerance of sole to hypoxia when fish were fed a lipid-rich diet (Zambonino-Infante et al., 2013). Statistical analysis revealed 801 genes (FDR $<10 \%$, with a p-value of 0.005 ) regulated by the dietary lipid content, whatever the oxygenation conditions (table 1; figure 1). These included 424 genes down-expressed and 377 genes up-expressed in fish fed the high lipid diet (additional file 3). Interestingly, we found only 200 genes with expression differentially affected by hypoxia depending on the dietary lipid content. It is noteworthy that gene ontologies related to macromolecule biosynthesis and protein folding, mitochondrial ATP synthesis and defense activity were significantly enriched within genes regulated by diet condition whatever the oxygenation conditions (table 4).

\subsubsection{Regulation of glucose metabolism}

We found that hepatic nuclear factor 6 (ONECUT1) was stimulated in fish fed the high fat diet (table 5). It is known that ONECUT1 is involved in the regulation of the transcription of gluconeogenic enzymes such as glucose-6-phosphatase (G6PC) (Streeper et al. 2001). In line with this, we also observed a stimulation of TCA enzymes involved in gluconeogeneis (MDH1 and $\mathrm{MDH} 2$ ) in the same dietary group (table 5). Together with all the mechanisms involved in high-fat diet-induced diabetes, this result could explain the higher glycemia rate observed in fish fed the high lipid diet (Zambonino-Infante et al., 2013).

\subsubsection{Stimulation of an ATP-expensive anabolic process and aerobic ATP synthesis}

Analysis of GO revealed that up-regulated genes were also related to protein biosynthesis and folding (61 genes, see table 5). The stimulation of genes involved in protein synthesis and maturation could be due to an increased need for lipoproteins, required to transport the overload of dietary lipids (Lim et al. 2009; Yildirim-Aksoy et al. 2009). This result contrasts with data obtained in mice fed a high fat diet, where a down-regulation of protein synthesis was observed (Deldicque et al. 2010; Oyadomari et al. 2008). This discrepancy may be likened to the low lipid tolerance of flatfish species (Borges et al. 2009). As mentioned by Zambonino-Infante et al. (2013), the relative difficulty that sole have at handling high dietary lipid contents may have a metabolic cost. In agreement with this idea, the present study revealed some up-regulated genes involved in oxidative phosphorylation and ATP biosynthesis (NDUFs and ATP synthases, table 5). Globally, around $10 \%$ of the genes up and down-regulated by hypoxia factor were found to be down and up-regulated by lipid factor, respectively (figure 1); GO over-represented within those genes being related to mitochondria and hydrogen ion transporter activity (table 6). These data suggest an increased activity of aerobic ATP production in response to high-fat feeding in common sole, probably through the positive regulation of fatty acid beta-oxidation and the TCA pathway.

The stimulation of ATP-expensive anabolic process, supported by the lower rates although not significant ( $p=0.07$ ) of AMPK $\alpha$ phosphorylation (figure 2 ), goes against the principle that hypoxic fish should save oxygen. These regulations were unrelated to the oxygen conditions and could therefore explain the impaired tolerance to hypoxia in fish fed the high lipid diet.

\subsubsection{Low significant hypoxia $x$ lipid interaction on the hepatic transcriptome}

Our statistical analysis revealed only a slight interaction between hypoxia and lipid factors on the hepatic transcriptome (i.e., 200 genes, see table 1 and additional file 3). However, biological data mining resulting from this analysis is ambiguous since FDR is expected to be 
around $30 \%$ using a p-value of 0.005 . Moreover, no gene ontology was enriched among the 200 genes.

\subsubsection{Impact of dietary lipid content on defence activities}

Finally, our transcriptomic data revealed that feeding fish a high lipid content diet downregulated genes associated with blood coagulation, immune response and homeostasis (table 5). These data confirm earlier studies that demonstrated an effect of the dietary lipid content on the fish immune system, particularly on complement activity (Geay et al. 2011). Together with the impact of high lipid ingestion on the energy metabolism of common sole, this last result suggests deleterious effects on the processes of defence, and further undermines the tolerance of this fish species to lipids.

\section{Conclusion}

The present data reveal new relevant information about the short term effect of acute hypoxia on the fish liver transcriptome. In particular, the data highlight a finely tuned regulation of different biological processes, including metabolic pathways and UPR response, resulting in metabolic depression. Our results revealed that the regulation of these processes could be related to the stimulation of several transcription factors, explaining the large-scale regulation of the hepatic transcriptome. Moreover, our data provide valuable insight on the influence of high lipid consumption on liver metabolic pathways. While several other tissues (i.e., muscle, heart and brain), as well as post-transcriptomic levels of regulations, are certainly affected by lipid content in diet, we can hypothesize that the regulations induced by high lipid diets in liver are detrimental to the energy-saving required for cellular homeostasis under hypoxia. This effect could partly contribute to explaining why fish fed high level of fat exhibited lower tolerance to hypoxia compared to those fed a low lipid diet. It is indeed accepted that hypoxia-tolerance is largely based on an organism's ability to down-regulate ATP production and consumption in a coordinated way during energy limiting conditions (Krumschnabel et al. 2000). It is suggested that climate warming will induce changes on zooplankton communities and especially lipid-storing species, which would affect the lipid flux of the entire system (Lee et al. 2006). Assessing the molecular mechanisms underlying the effects of dietary lipid content on hypoxia tolerance in common sole is crucial for a better understanding the effects that global change will have on the physiology of this specific taxon, on its life-traits and the possible consequences in term of population.

\section{Acknowledgements}

We thank P. Quazuguel for his excellent assistance in maintaining the fish and C. Huelvan and M.M. Le Gall for their assistance with sampling. We are also grateful to C. Quere for their assistance with AMPK analysis and to $\mathrm{H}$. McCombie for English language editing. This work was supported by the SINGER-POLIGNAC foundation. 


\section{References}

Adachi, H. \& Tsujimoto, M. (2002). FEEL-1, a novel scavenger receptor with in vitro bacteriabinding and angiogenesis-modulating activities. Journal of Biological Chemistry, 277: 34264-34270.

Almeida-Val, V.M.F., Chipari-Gomes, A.R. \& Lopes, N.P. (2006). Metabolic and physiological adjustments to low oxygen and high temperature in fishes of the Amazon. IN A. L. Val , V.M.F.A.-V., D. J. Randall (Ed.) The Physiology of Tropical Fishes. London: Elsevier.

Ameri, K. \& Harris, A.L. (2008). Activating transcription factor 4. International Journal of Biochemistry \& Cell Biology, 40: 14-21.

Bickler, P.E. \& Buck, L.T. (2007). Hypoxia tolerance in reptiles, amphibians, and fishes: Life with variable oxygen availability. Annual Review of Physiology.

Borges, P., Oliveira, B., Casal, S., Dias, J., Conceicao, L. \& Valente, L.M.P. (2009). Dietary lipid level affects growth performance and nutrient utilisation of Senegalese sole (Solea senegalensis) juveniles. British Journal of Nutrition, 102: 1007-1014.

Cannas, M., Bayle, A., Wing, O.L.C., Domenici, P. \& Lefrancois, C. (2007). Behaviour and physiology of the startle response in common sole (Solea solea) exposed to hypoxia. Comparative Biochemistry and Physiology a-Molecular \& Integrative Physiology, 146: S85-S85.

Chandel, N.S., Maltepe, E., Goldwasser, E., Mathieu, C.E., Simon, M.C. \& Schumacker, P.T. (1998). Mitochondrial reactive oxygen species trigger hypoxia-induced transcription. Proceedings of the National Academy of Sciences of the United States of America, 95: 11715-11720.

Corporeau, C. \& Auffret, M. (2003). In situ hybridisation for flow cytometry: a molecular method for monitoring stress-gene expression in hemolymph cells of oysters. Aquat Toxicol. 64:427-435.

Cummins, E.P. \& Taylor, C.T. (2005). Hypoxia-responsive transcription factors. Pflugers Archiv-European Journal of Physiology, 450: 363-371.

Dalla Via, J., Vandenthillart, G., Cattani, O. \& Cortesi, P. (1997). Environmental versus functional hypoxia/anoxia in sole Solea solea: The lactate paradox revisited. Marine Ecology-Progress Series, 154: 79-90.

Dalla Via, J., Vandenthillart, G., Cattani, O. \& Dezwaan, A. (1994). Influence of long-term hypoxia exposure on the energy-metabolism of solea-solea .2. intermediary metabolism in blood, liver and muscle. Marine Ecology-Progress Series, 111: 17-27.

Deldicque, L., Cani, P.D., Philp, A., Raymackers, J.-M., Meakin, P.J., Ashford, M.L.J., Delzenne, N.M., Francaux, M. \& Baar, K. (2010). The unfolded protein response is activated in skeletal muscle by high-fat feeding: potential role in the downregulation of protein synthesis. American Journal of Physiology-Endocrinology and Metabolism, 299: E695-E705.

Everett, M.V., Antal, C.E. \& Crawford, D.L. (2012). The effect of short-term hypoxic exposure on metabolic gene expression. Journal of Experimental Zoology Part a-Ecological Genetics and Physiology, 317A: 9-23.

Geay, F., Ferraresso, S., Zambonino-Infante, J.L., Bargelloni, L., Quentel, C., Vandeputte, M., Kaushik, S., Cahu, C.L. \& Mazurais, D. (2011). Effects of the total replacement of fish-based diet with plant-based diet on the hepatic transcriptome of two European sea bass (Dicentrarchus labrax) half-sibfamilies showing different growth rates with the plant-based diet. Bmc Genomics, 12.

Gracey, A.Y., Troll, J.V. \& Somero, G.N. (2001). Hypoxia-induced gene expression profiling in the euryoxic fish Gillichthys mirabilis. Proceedings of the National Academy of Sciences of the United States of America, 98: 1993-1998.

Hardie, D.G. (2008). AMPK: a key regulator of energy balance in the single cell and the whole organism. Int $J$ Obes (Lond). 32 Suppl 4:S7-12. 
Harding, H.P., Zhang, Y.H., Zeng, H.Q., Novoa, I., Lu, P.D., Calfon, M., Sadri, N., Yun, C., Popko, B., Paules, R., Stojdl, D.F., Bell, J.C., Hettmann, T., Leiden, J.M. \& Ron, D. (2003). An integrated stress response regulates amino acid metabolism and resistance to oxidative stress. Molecular Cell, 11: 619-633.

Ho, K.J., Do, N.L., Otu, H.H., Dib, M.J., Ren, X., Enjyoji, K., Robson, S.C., Terwilliger, E.F. \& Karp, S.J. (2010). Tob1 is a constitutively expressed repressor of liver regeneration. Journal of Experimental Medicine, 207: 1197-1208.

Hochachka, P.W., Buck, L.T., Doll, C.J. \& Land, S.C. (1996). Unifying theory of hypoxia tolerance: Molecular metabolic defense and rescue mechanisms for surviving oxygen lack. Proceedings of the National Academy of Sciences of the United States of America, 93: 9493-9498.

Ichikawa-Shindo, Y., Sakurai, T., Kamiyoshi, A., Kawate, H., Linurna, N., Yoshizawa, T., Koyama, T., Fukuchi, J., Limuro, S., Moriyama, N., Kawakami, H., Murata, T., Kangawa, K., Nagai, R. \& Shindo, T. (2008). The GPCR modulator protein RAMP2 is essential for angiogenesis and vascular integrity. Journal of Clinical Investigation, 118: 29-39.

Jaeger, R., Bertrand, M.J.M., Gorman, A.M., Vandenabeele, P. \& Samali, A. (2012). The unfolded protein response at the crossroads of cellular life and death during endoplasmic reticulum stress. Biology of the Cell, 104: 259-270.

Jibb, L.A. \& Richards, J.G. (2008). AMP-activated protein kinase activity during metabolic rate depression in the hypoxic goldfish, Carassius auratus. Journal of Experimental Biology, 211: 3111-3122.

Ju, Z., Wells, M.C., Heater, S.J. \& Walter, R.B. (2007). Multiple tissue gene expression analyses in Japanese medaka (Oryzias latipes) exposed to hypoxia. Comparative Biochemistry and Physiology C-Toxicology \& Pharmacology, 145: 134-144.

Kajimura, S., Aida, K. \& Duan, C.M. (2005). Insulin-like growth factor-binding protein-1 (IGFBP-1) mediates hypoxia-induced embryonic growth and developmental retardation. Proceedings of the National Academy of Sciences of the United States of America, 102: 1240-1245.

Kamaid, A. \& Giraldez, F. (2008). Btg1 and Btg2 gene expression during early chick development. Developmental Dynamics, 237: 2158-2169.

Kim, I., Xu, W. \& Reed, J.C. (2008). Cell death and endoplasmic reticulum stress: disease relevance and therapeutic opportunities. Nature Reviews Drug Discovery, 7: 10131030.

Krumschnabel, G., Schwarzbaum, P.J., Lisch, J., Biasi, C. \& Wieser, W. (2000). Oxygendependent energetics of anoxia-tolerant and anoxia-intolerant hepatocytes. Journal of Experimental Biology, 203: 951-959.

Leaver, M.J., Villeneuve, L.A., Obach, A., Jensen, L., Bron, J.E., Tocher, D.R. \& Taggart, J.B. (2008). Functional genomics reveals increases in cholesterol biosynthetic genes and highly unsaturated fatty acid biosynthesis after dietary substitution of fish oil with vegetable oils in Atlantic salmon (Salmo salar). BMC Genomics, 9:299-315.

Le Pape, O., Chauvet, F., Mahevas, S., Lazure, P., Guerault, D. \& Desaunay, Y. (2003). Quantitative description of habitat suitability for the juvenile common sole (Solea solea, L.) in the Bay of Biscay (France) and the contribution of different habitats to the adult population. Journal of Sea Research, 50: 139-149.

Lee, R.F., Hagen, W. \& Kattner, G. (2006). Lipid storage in marine zooplankton. Marine Ecology-Progress Series, 307: 273-306.

Leveelahti, L., Leskinen, P., Leder, E.H., Waser, W. \& Nikinmaa, M. (2011). Responses of threespine stickleback (Gasterosteus aculeatus, L) transcriptome to hypoxia. Comparative Biochemistry and Physiology D-Genomics \& Proteomics, 6: 370-381.

Lim, C., Yildirim-Aksoy, M., Li, M.H., Welker, T.L. \& Klesius, P.H. (2009). Influence of dietary levels of lipid and vitamin $E$ on growth and resistance of Nile tilapia to Streptococcus iniae challenge. Aquaculture, 298: 76-82.

Liu, F. \& Roth, R.A. (1995). GRB-IR - A SH2-DOMAIN-CONTAINING PROTEIN THAT BINDS TO THE INSULIN-RECEPTOR AND INHIBITS ITS FUNCTION. Proceedings 
of the National Academy of Sciences of the United States of America, 92: 1028710291.

Lopez-Barneo, J., Nurse, C.A., Nilsson, G.E., Buck, L.T., Gassmann, M. \& Bogdanova, A.Y. (2010). First Aid Kit for Hypoxic Survival: Sensors and Strategies. Physiological and Biochemical Zoology, 83: 753-763.

Luo, S.Z., Baumeister, P., Yang, S.J., Abcouwer, S.F. \& Lee, A.S. (2003). Induction of Grp78/BiP by translational block - Activation of the Grp78 promoter by ATF4 through an upstream ATF/CRE site independent of the endoplasmic reticulum stress elements. Journal of Biological Chemistry, 278: 37375-37385.

Ma, Y.J. \& Hendershot, L.M. (2004). Herp is dually regulated by both the endoplasmic reticulum stress-specific branch of the unfolded protein response and a branch that is shared with other cellular stress pathways. Journal of Biological Chemistry, 279: 13792-13799.

Mandic, M., Todgham, A.E. \& Richards, J.G. (2009). Mechanisms and evolution of hypoxia tolerance in fish. Proceedings of the Royal Society B-Biological Sciences, 276: 735744.

Mansfield, K.D., Guzy, R.D., Pan, Y., Young, R.M., Cash, T.P., Schumacker, P.T. \& Simon, M.C. (2005). Mitochondrial dysfunction resulting from loss of cytochrome c impairs cellular oxygen sensing and hypoxic HIF-alpha activation. Cell Metabolism, 1: 393399.

Marinsky, C.A., Houston, A.H. \& Murad, A. (1990). EFFECT OF HYPOXIA ON HEMOGLOBIN ISOMORPH ABUNDANCES IN RAINBOW-TROUT, SALMOGAIRDNERI. Canadian Journal of Zoology-Revue Canadienne De Zoologie, 68: 884888.

Martinez, M.L., Landry, C., Boehm, R., Manning, S., Cheek, A.O. \& Rees, B.B. (2006). Effects of long-term hypoxia on enzymes of carbohydrate metabolism in the Gulf killifish, Fundulus grandis. Journal of Experimental Biology, 209: 3851-3861.

Mckenzie, D.J., Lund, I. \& Pedersen, P.B. (2008). Essential fatty acids influence metabolic rate and tolerance of hypoxia in Dover sole (Solea solea) larvae and juveniles. Marine Biology, 154: 1041-1051.

Mihaylova, M.M. \& Shaw, R.J. (2011). The AMPK signalling pathway coordinates cell growth, autophagy and metabolism. Nature Cell Biology, 13: 1016-1023.

Min, S.H., Mackenzie, D.D.S., Breier, B.H., Mccutcheon, S.N. \& Gluckman, P.D. (1996). Responses of young energy-restricted sheep to chronically administered insulin-like growth factor I (IGF-I): Evidence that IGF-I suppresses the hepatic growth hormone receptor. Endocrinology, 137: 1129-1137.

Nikinmaa, M. \& Rees, B.B. (2005). Oxygen-dependent gene expression in fishes. American Journal of Physiology-Regulatory Integrative and Comparative Physiology, 288: R1079-R1090.

Oyadomari, S., Harding, H.P., Zhang, Y., Oyadomari, M. \& Ron, D. (2008). Dephosphorylation of translation initiation factor 2 alpha enhances glucose tolerance and attenuates hepatosteatosis in mice. Cell Metabolism, 7: 520-532.

Richards, J.G. (2009). Metabolic and molecular responses of fish to hypoxia. IN Richards, J.G., Farrell, A.P. \& Brauner, C.J. (Eds.) Hypoxia. San Diego: Elsevier.

Richards, J.G. (2011). Physiological, behavioral and biochemical adaptations of intertidal fishes to hypoxia. Journal of Experimental Biology, 214: 191-199.

Russell, K.S., Stern, D.F., Polverini, P.J. \& Bender, J.R. (1999). Neuregulin activation of ErbB receptors in vascular endothelium leads to angiogenesis. American Journal of Physiology-Heart and Circulatory Physiology, 277: H2205-H2211.

Semenza, G.L., Jiang, B.H., Leung, S.W., Passantino, R., Concordet, J.P., Maire, P. \& Giallongo, A. (1996). Hypoxia response elements in the aldolase A, enolase 1, and lactate dehydrogenase $A$ gene promoters contain essential binding sites for hypoxiainducible factor 1. Journal of Biological Chemistry, 271: 32529-32537. 
Stankiewicz, P., Sen, P., Bhatt, S.S., Storer, M., Xia, Z., Bejjani, B.A., Ou, Z., Wiszniewska, J., Driscoll, D.J., Bolivar, J., Bauer, M., Zackai, E.H., Mcdonald-Mcginn, D., Nowaczyk, M.M.J., Murray, M., Shaikh, T.H., Martin, V., Tyreman, M., Simonic, I., Willatt, L., Paterson, J., Mehta, S., Rajan, D., Fitzgerald, T., Gribble, S., Prigmore, E., Patel, A., Shaffer, L.G., Carter, N.P., Cheung, S.W., Langston, C. \& Shaw-Smith, C. (2009). Genomic and Genic Deletions of the FOX Gene Cluster on 16q24.1 and Inactivating Mutations of FOXF1 Cause Alveolar Capillary Dysplasia and Other Malformations. American Journal of Human Genetics, 84: 780-791.

Streeper, R.S., Hornbuckle, L.A., Svitek, C.A., Goldman, J.K., Oeser, J.K. \& O'brien, R.M. (2001). Protein kinase A phosphorylates hepatocyte nuclear factor-6 and stimulates glucose-6-phosphatase catalytic subunit gene transcription. Journal of Biological Chemistry, 276: 19111-19118.

Tagliavacca, L., Caretti, A., Bianciardi, P. \& Samaja, M. (2012). In vivo up-regulation of the unfolded protein response after hypoxia. Biochimica Et Biophysica Acta-General Subjects, 1820: 900-906.

Tazuke, S.I., Mazure, N.M., Sugawara, J., Carland, G., Faessen, G.H., Suen, L.F., Irwin, J.C., Powell, D.R., Giaccia, A.J. \& Giudice, L.C. (1998). Hypoxia stimulates insulinlike growth factor binding protein 1 (IGFBP-1) gene expression in HepG2 cells: A possible model for IGFBP-1 expression in fetal hypoxia. Proceedings of the National Academy of Sciences of the United States of America, 95: 10188-10193.

Van Den Beucken, T., Magagnin, M.G., Jutten, B., Seigneuric, R., Lambin, P., Koritzinsky, M. \& Wouters, B.G. (2011). Translational control is a major contributor to hypoxia induced gene expression. Radiotherapy and Oncology, 99: 379-384.

Van Den Thillart, G., Dalla Via, J., Vitali, G. \& Cortesi, P. (1994). INFLUENCE OF LONGTERM HYPOXIA EXPOSURE ON THE ENERGY-METABOLISM OF SOLEA-SOLEA .1. CRITICAL O-2 LEVELS FOR AEROBIC AND ANAEROBIC METABOLISM. Marine Ecology Progress Series, 104: 109-117.

Wang, A.H., Nomura, M., Patan, S. \& Ware, J.A. (2002). Inhibition of protein kinase C alpha prevents endothelial cell migration and vascular tube formation in vitro and myocardial neovascularization in vivo. Circulation Research, 90: 609-616.

Wright, P.A., Perry, S.F. \& Moon, T.W. (1989). Regulation of hepatic Gluconeogensis and glycogenolysis by cathecholamines in rainbow-trout during environmental hypoxia. Journal of Experimental Biology, 147: 169-188.

Wu, R.S.S. (2002). Hypoxia: from molecular responses to ecosystem responses. Marine Pollution Bulletin, 45: 35-45.

Yen, L., Benlimame, N., Nie, Z.R., Xiao, D.Z., Wang, T.Q., Al Moustafa, A.E., Esumi, H., Milanini, J., Hynes, N.E., Pages, G. \& Alaoui-Jamali, M.A. (2002). Differential regulation of tumor angiogenesis by distinct ErbB homo- and heterodimers. Molecular Biology of the Cell, 13: 4029-4044.

Yildirim-Aksoy, M., Lim, C., Shelby, R. \& Klesius, P.H. (2009). Increasing Fish Oil Levels in Commercial Diets Influences Hematological and Immunological Responses of Channel Catfish, Ictalurus punctatus. Journal of the World Aquaculture Society, 40: 76-86.

Zambonino-Infante, J., Claireaux, G., Ernande, B., Jolivet, A., Quazuguel, P., Sévère, A., Huelvan, C. \& Mazurais, D. (2013). Hypoxia tolerance of common sole juveniles depends on dietary regime and temperature at the larval stage: Evidence for environmental conditioning. Proc $R$ Soc B. in press. doi: 10.1098/rspb.2012.3022.

Zimrin, A.B., Pepper, M.S., Mcmahon, G.A., Nguyen, F., Montesano, R. \& Maciag, T. (1996). An antisense oligonucleotide to the notch ligand jagged enhances fibroblast growth factor-induced angiogenesis in vitro. Journal of Biological Chemistry, 271: 3249932502. 


\section{Tables}

Table 1: Number of genes regulated by the factors "hypoxia" and "lipid", and responding to the "hypoxia $\times$ lipid" interaction, as revealed by two-way ANOVA on transcriptomic data.

\begin{tabular}{lccc}
\hline \hline 4.1. Factors & 4.2. Hypoxia & 4.3. Lipid & $\begin{array}{c}\text { 4.4. Hypoxia } \\
\times \text { Lipid }\end{array}$ \\
\hline \hline $\begin{array}{l}\text { 4.5. Number of } \\
\text { regulated genes }\end{array}$ & $\begin{array}{c}\text { 4.6. } 1202 \\
\text { up: } 514 \text { down: } 688\end{array}$ & $\begin{array}{c}\text { 4.7. } 801 \\
\text { up: } 424 \text { down: } 377\end{array}$ & 4.8. 200 \\
\hline \hline
\end{tabular}

Table 2: Gene Ontologies over-represented among genes regulated by the factor "hypoxia"

\begin{tabular}{llcc}
\hline \hline System & \multicolumn{1}{c}{ Gene Ontology } & Number of genes & $\begin{array}{c}\text { Corrected } \\
\text { (Benjamini) } \\
\text { p-value }\end{array}$ \\
\hline \hline Biological process & carbohydrate metabolism & 40 & 0.04 \\
& glucose metabolism & 16 & 0.03 \\
& energy pathways & 31 & 0.03 \\
Molecular function & oxidoreductase activity & 56 & 0.01
\end{tabular}


Table 3: Summary of selected down- and up-regulated transcripts in the liver of Solea solea 90 min after the beginning of the hypoxic challenge

\begin{tabular}{ccc}
\hline \hline Transcripts & Official & p-value \\
& gene \\
name
\end{tabular}

Down-regulated

ATP synthesis-coupled electron transport

NADH dehydrogenase 1 beta subcomplex subunit 2

6.92E-4

NADH dehydrogenase 1 beta subcomplex subunit 8

NADH-ubiquinone oxidoreductase $75 \mathrm{kDa}$ subunit

$\mathrm{NADH}$ dehydrogenase flavoprotein 2

NDUFB2

NDUFB8 4.63E-4

NDUFS1 0.0028

NDUFV2 $\quad 0.0026$

\section{TCA intermediate metabolism}

ATP-citrate synthase
Glutamate decarboxylase 1
Isocitrate dehydrogenase
NADP-dependent malic enzyme

Glycolysis

Eno1 protein

Glucose-6-phosphate 1-dehydrogenase

Hexokinase-2

L-lactate dehydrogenase $A$

L-lactate dehydrogenase $B$

6-phosphofructokinase

6-phosphogluconate dehydrogenase

Phosphoglycerate kinase 1

Pyruvate kinase isozyme M1/M2

Transaldolase

\section{Lipid biosynthesis}

Arachidonate 12-lipoxygenase

Delta(14)-sterol reductase

Squalene monooxygenase

Acyl-CoA desaturase

Lathosterol oxidase

Phosphatidylinositol $\mathrm{N}$-acetylglucosaminyl transferase $\mathrm{H}$

Phosphatidylinositol $N$-acetylglucosaminyl transferase $B$

Ethanolamine-phosphate cytidylyltransferase

Methionine adenosyltransferase 2 subunit beta

Lanosterol synthase

3-keto-steroid reductase

3-hydroxy-3-methylglutaryl-coenzyme A reductase

Fatty acid synthase

7-dehydrocholesterol reductase

Arachidonate 5-lipoxygenase

$\begin{array}{cc}\text { ACLY } & 2.63 \mathrm{E}-4 \\ \text { GAD1 } & 6.03 \mathrm{E}-4 \\ \text { IDH1 } & 0.0014 \\ \text { ME1 } & 0.0047\end{array}$

$\begin{array}{cc}\text { ENO1 } & 4.64 \mathrm{E}-9 \\ \text { G6PD } & 2.46 \mathrm{E}-9 \\ \text { HK2 } & 1.37 \mathrm{E}-4 \\ \text { LDHA } & 4.20 \mathrm{E}-4 \\ \text { LDHB } & 7.22 \mathrm{E}-5 \\ \text { PFKL } & 0.0031 \\ \text { PGD } & 1.50 \mathrm{E}-7 \\ \text { PGK1 } & 3.93 \mathrm{E}-5 \\ \text { PKM2 } & 0.0027 \\ \text { TALDO1 } & 6.53 \mathrm{E}-7\end{array}$

$\begin{array}{cc}\text { ALOX12 } & 8.42 \mathrm{E}-4 \\ \text { TM7SF2 } & 0.0041 \\ \text { SQLE } & 1.24 \mathrm{E}-4 \\ \text { SCD } & 1.93 \mathrm{E}-6 \\ \text { SC5DL } & 0.0049 \\ \text { PIGH } & 2.77 \mathrm{E}-4 \\ \text { PIGB } & 1.75 \mathrm{E}-4 \\ \text { PCYT2 } & 0.0015 \\ \text { MAT2B } & 5.28 \mathrm{E}-4 \\ \text { LSS } & 0.0048 \\ \text { HSD17B7 } & 0.0037 \\ \text { HMGCR } & 4.20 \mathrm{E}-6 \\ \text { FASN } & 2.45 \mathrm{E}-6 \\ \text { DHCR7 } & 2.28 \mathrm{E}-5 \\ \text { ALOX5 } & 3.49 \mathrm{E}-4\end{array}$


ATP-citrate synthase

ACLY

2.26E-4

Acetyl-CoA carboxylase $1 \mathrm{~s}$

ACACA

0.0029

\section{Protein folding}

$T$-complex protein 1 subunit gamma

T-complex protein 1 subunit epsilon

DnaJ homolog subfamily $B$ member 2

$\begin{array}{cc}\text { CCT3 } & 0.0027 \\ \text { CCT5 } & 1.33 \mathrm{E}-4 \\ \text { DNAJB2 } & 7.88 \mathrm{E}-4 \\ \text { ERO1L } & 0.0019 \\ \text { FKBP3 } & 0.0020 \\ \text { HSPA8 } & 0.0012 \\ \text { HSPE1 } & 4.58 \mathrm{E}-4 \\ \text { TBCD } & 0.0014 \\ \text { TSC2 } & 1.72 \mathrm{E}-5\end{array}$

ERO1-like protein alpha

Peptidyl-prolyl cis-trans isomerase FKBP3

Heat shock cognate $71 \mathrm{kDa}$ protein

$10 \mathrm{kDa}$ heat shock protein, mitochondrial

Tubulin-specific chaperone $D$

Tuberin

\section{Endoplasmic reticulum}

Cytochrome P450 2J2

CYP2J2

$2.25 \mathrm{E}-4$

Cytochrome P450 2S1

CYP2S1 9.65E-5

7-alpha-hydroxycholest-4-en-3-one 12-alpha-hydroxylase

CYP8B1

0,0037

7-dehydrocholesterol reductase

ERO1-like protein alpha

3-hydroxy-3-methylglutaryl-coenzyme A reductase

Gamma-soluble NSF attachment protein

Protein-L-isoaspartate( $D$-aspartate) O-methyltransferase

Phosphatidylinositol $N$-acetylglucosaminyl transferase $B$

DHCR7

$2.28 \mathrm{E}-5$

ERO1L

0.0019

HMGCR

$4.20 \mathrm{E}-5$

$N A P G$

0,0035

Phosphatidylinositol $\mathrm{N}$-acetylglucosaminyl transferase $\mathrm{H}$

PCMT1

4.66E-4

$P I G B$

$1.75 \mathrm{E}-4$

Proteolipid protein 2

Proteasome subunit alpha 2

Proteasome subunit beta 4

Proteasome subunit beta 5

PIGH

2.77E-4

PLP2

$3.62 \mathrm{E}-5$

PSMA2

6.69E-5

PSMB4

3.26E-6

Proteasome subunit beta 7

PSMB5

0,0030

Proteasome subunit delta 5

PSMB7

$3.29 \mathrm{E}-5$

Proteasome activator complex subunit 1

PSMD5

$1.15 \mathrm{E}-6$

Reticulon-4

PSME 1

4.40E-4

Lathosterol oxidase

RTN4

$8.08 \mathrm{E}-10$

Acyl-CoA desaturase

$S C 5 D L$

0.0049

Delta(14)-sterol reductase

$S C D$

1.93E-6

TM7SF2 $\quad 0,0042$

\section{Up-regulated}

\section{Gluconeogenesis pathway}

Phosphoenolpyruvate carboxykinase

$\begin{array}{ll}\text { PCK1 } & 5.86 \mathrm{E}-4 \\ \text { G6PC } & 1.32 \mathrm{E}-4\end{array}$

Glucose-6-phosphatase

G6PC

0.0018

Kynurenine/alpha-aminoadipate aminotransferase

$\begin{array}{ll}\text { AADAT } & 0.0018 \\ \text { GOT1 } & 5.63 \mathrm{E}-5 \\ \text { OAT } & 1.09 \mathrm{E}-4 \\ \text { HAL } & 8.58 \mathrm{E}-7 \\ \text { TAT } & 2.10 \mathrm{E}-4\end{array}$

Aspartate aminotransferase

Ornithine aminotransferase

1.09E-4

Histidine ammonia lyase

TAT

2.10E-4

\section{Regulation of cell growth and proliferation}


Insulin-like growth factor-binding protein 1

Growth factor receptor-bound protein 10

Dual specificity protein phosphatase 1

Dual specificity protein phosphatase 6

Transducer of erbB-2 1

$B$-cell translocation gene 1 protein

\section{Vessel development}

Stabilin-1

Growth factor receptor-bound protein 10

Receptor tyrosine-protein kinase erbB-4

Epidermal growth factor receptor

Protein kinase $C$ alpha type

Platelet-derived growth factor receptor beta

Forkhead box protein F1

Protein jagged-1

Receptor activity-modifying protein 2

\section{Sulfur amino acid biosynthesis}

Cystathionine beta-synthase

Cystathionine gamma-lyase

Gamma-glutamyltranspeptidase 1

\section{Transcription factors}

Transcription factor jun-B

Proto-oncogene c-Fos

Early growth response protein 1

CCAAT/enhancer-binding protein delta

Hypoxia-inducible factor 3-alpha

Cyclic AMP-responsive element-binding protein 1

Activating transcription factor 7 -interacting protein 1

$\begin{array}{ll}\text { IGFBP-1 } & 6.94 \mathrm{E}-6 \\ \text { GRB10 } & 2.88 \mathrm{E}-4 \\ \text { DUSP1 } & 0.0012 \\ \text { DUSP6 } & 0.0014 \\ \text { TOB1 } & 0.0025 \\ \text { BTG-1 } & 5.69 \mathrm{E}-6\end{array}$

STAB1 $\quad 0.0017$

GRB10 2.88E-4

ERBB4 $\quad 0.0049$

EGFR 7.02E-4

PRKCA 1.55E-4

PDGFRB $\quad 3.33 \mathrm{E}-4$

FOXF1 0.0029

JAG1 2.36E-4

RAMP2 1.80E-4

$\begin{array}{cr}\text { CBS } & 2.38 \mathrm{E}-8 \\ \text { CTH } & 8.29 \mathrm{E}-5 \\ \text { GGT1 } & 7.59 \mathrm{E}-4\end{array}$

$\begin{array}{ll}\text { JUNB } & 1.69 \mathrm{E}-8 \\ \text { FOS } & 3.36 \mathrm{E}-4 \\ \text { EGR1 } & 1.67 \mathrm{E}-4 \\ \text { CEBPD } & 0.0033 \\ \text { HIF3 } & 1.56 \mathrm{E}-4 \\ \text { CREB1 } & 0.0037 \\ \text { ATF7IP } & 8.47 \mathrm{E}-4\end{array}$

\section{Other}

Cationic amino acid transporter 3

SLC7A3 $\quad 0.0031$

78 kDa glucose-regulated protein

HSPA5 1.80E-4

GHR $\quad 3.78 \mathrm{E}-4$

Growth hormone receptor

endoplasmic reticulum-resident HERPUD1

$9.46 \mathrm{E}-4$

ubiquitin-like domain member 1 protein 
Table 4: Gene Ontologies over-represented among genes regulated by high dietary lipid

\begin{tabular}{llcc}
\hline \hline \multirow{2}{*}{ System } & \multicolumn{1}{c}{ Gene Ontology } & $\begin{array}{c}\text { Number } \\
\text { genes }\end{array}$ & $\begin{array}{c}\text { Corrected } \\
\text { of }\end{array}$ \\
& & $\begin{array}{c}\text { (Benjamini) } \\
\text { p-value }\end{array}$ \\
\hline \hline Biological process & protein biosynthesis & 52 & $2,06 \mathrm{E}-06$ \\
& complement activation & 8 & $2,50 \mathrm{E}-03$ \\
& hydrogen transport & 11 & 0.03 \\
& protein folding & 15 & 0.04 \\
bellular componen & blood coagulation & 12 & 0.04 \\
& ribosome & 39 & $1,02 \mathrm{E}-06$ \\
& cytosol & 46 & $2,06 \mathrm{E}-06$ \\
& proton-transporting ATP synthase complex & 6 & $2,50 \mathrm{E}-03$ \\
Molecular function & mitochondrial inner membrane & 14 & 0.04 \\
& hydrogen ion transporter activity & 24 & $2,06 \mathrm{E}-06$ \\
& RNA binding & 37 & 0.01 \\
& defense/immunity protein activity & 11 & 0.03
\end{tabular}

Table 5: Summary of selected transcripts down and up-regulated in the liver of Solea solea by high dietary lipid

\begin{tabular}{lll}
\hline \hline \multicolumn{1}{c}{ Transcripts } & \multicolumn{1}{c}{$\begin{array}{c}\text { Official } \\
\text { gene name }\end{array}$} & p-value \\
\hline \hline Down-regulated & & \\
& & \\
Immune response & & \\
Beta-2-glycoprotein 1 & APOH & $4.68 \mathrm{E}-4$ \\
Attractin & ATRN & 0.0026 \\
Complement C1r subcomponent & C1R & $5.04 \mathrm{E}-4$ \\
Complement C3 & C3 & $3.95 \mathrm{E}-5$ \\
Complement C5 & C5 & 0.0036 \\
Complement component C8 alpha chain & C8A & 0.0049 \\
Complement component C8 gamma chain & C8G & $8.36 \mathrm{E}-4$ \\
Immunoglobulin-binding protein 1 & IGBP1 & $9.03 \mathrm{E}-4$ \\
Lysozyme C & LYZ & 0.0028 \\
Mannan-binding lectin serine protease 1 & MASP1 & $2.14 \mathrm{E}-7$ \\
Phosphatidylinositol 3-kinase regulatory subunit alpha & PIK3R1 & 0.0046 \\
Parathymosin & PTMS & $8.06 \mathrm{E}-4$ \\
E-selectin & SELE & $3.04 \mathrm{E}-4$ \\
Alpha-1-antitrypsin & SERPINA1 & 0.0034 \\
Plasma protease C1 inhibitor & SERPING1 & $7.32 \mathrm{E}-4$
\end{tabular}




\section{Up-regulated}

\section{Protein biosynthesis}

Alanine--tRNA ligase, cytoplasmic

Cysteine--tRNA ligase, cytoplasmic

AARS

$7.18 \mathrm{E}-6$

T-complex protein 1 subunit beta

CARS

0,0034

Elongation factor 1-gamma

Elongation factor 2

CCT2

EEF1G $\quad 5.05 \mathrm{E}-5$

Translation initiation factor eIF-2B subunit epsilon

EEF2

0,0012

Eukaryotic translation initiation factor 2 subunit 2

EIF2B5

0.0028

Eukaryotic translation initiation factor 2 subunit 3

EIF2S2

0.0037

Eukaryotic translation initiation factor $5 A-1$

EIF2S3

$1.13 \mathrm{E}-5$

Bifunctional glutamate/proline--tRNA ligase

EIF5A

0.0028

Clustered mitochondria protein homolog

$39 S$ ribosomal protein L1, mitochondrial

$39 S$ ribosomal protein L15, mitochondrial

$39 S$ ribosomal protein L17, mitochondrial

$39 S$ ribosomal protein L4, mitochondrial

$39 S$ ribosomal protein L42, mitochondrial

EPRS

$1.98 \mathrm{E}-4$

KIAA0664

MRPL 1

0.0042

MRPL 15

MRPL17

0,0040

$28 S$ ribosomal protein S14, mitochondrial

MRPL4

$1.05 \mathrm{E}-5$

MRPL42

0,0011

$28 \mathrm{~S}$ ribosomal protein S2, mitochondrial

MRPS14

$28 S$ ribosomal protein S35, mitochondrial

MRPS2

MRPS35

Glycylpeptide N-tetradecanoyltransferase 1

NMT1

Serine/threonine-protein phosphatase $2 A 65 \mathrm{kDa}$ regulatory

Glutamine--tRNA ligase

PPP2R1B

0.0019

Glutamyl-tRNA(GIn) amidotransferase subunit $A$

$\begin{array}{ll}\text { QARS } & 2.92 \mathrm{E}-4 \\ \text { QRSL1 } & 1.70 \mathrm{E}-5\end{array}$

Arginine tRNA ligase, cytoplasmic

$60 S$ ribosomal protein $L 11$

60S ribosomal protein $L 13$

RARS

2.09E-4

RPL11

$5.63 \mathrm{E}-5$

$60 S$ ribosomal protein $L 18$ a

RPL13

$7.26 \mathrm{E}-5$

$60 S$ ribosomal protein $L 24$

$60 S$ ribosomal protein $L 27$

RPL18A

$1.77 \mathrm{E}-5$

RPL24 5.58E-4

$60 S$ ribosomal protein $L 3$

$60 S$ ribosomal protein $\angle 30$

RPL27

8.19E-4

RPL3

$7.41 \mathrm{E}-5$

$60 S$ ribosomal protein $\mathrm{L} 35 \mathrm{a}$

RPL30

0,0036

RPL35A $\quad 0.0029$

$60 S$ ribosomal protein $L 36$

$60 S$ ribosomal protein $L 38$

$60 S$ ribosomal protein $L 4$

$60 S$ ribosomal protein $L 7$

$60 S$ ribosomal protein $\angle 7 a$

$60 S$ ribosomal protein $L 8$

$40 S$ ribosomal protein $S 13$

RPL36

0,0013

RPL38

0,0017

RPL4

$1.37 \mathrm{E}-5$

RPL7

6.62E-4

RPL7A 5.86E-5

RPL8 1.44E-4

$40 S$ ribosomal protein $\$ 15 a$

RPS13

0,0030

40S ribosomal protein $\$ 19$

RPS15A

RPS19

$4.43 \mathrm{E}-4$

$40 S$ ribosomal protein $S 2$

RPS2

0,0012

$40 S$ ribosomal protein $S 21$

RPS21

$8.28 \mathrm{E}-4$

$40 S$ ribosomal protein 526

RPS26

0,0041

$40 S$ ribosomal protein S27a

RPS27A

0,0041

$40 S$ ribosomal protein S28

RPS28

$1.52 \mathrm{E}-4$

$40 S$ ribosomal protein S3

$40 S$ ribosomal protein 55

$40 S$ ribosomal protein 59

RPS3A

$5.82 \mathrm{E}-4$

RPS5

0,0014

RPS9

0,0033

Toll-like receptor 3

TLR3

$3.39 \mathrm{E}-4$
$2.95 \mathrm{E}-4$ 


\section{Protein folding}

T-complex protein 1 subunit beta

T-complex protein 1 subunit gamma

CCT2

CCT3

$5.17 \mathrm{E}-4$

$T$-complex protein 1 subunit delta

CCT4

$5.15 \mathrm{E}-6$

$T$-complex protein 1 subunit epsilon

CCT5

0.0012

$T$-complex protein 1 subunit eta

CCT7

$9.58 \mathrm{E}-8$

T-complex protein 1 subunit theta

CCT8

$6.34 \mathrm{E}-6$

DnaJ homolog subfamily $B$ member 2

DNAJB2

0.0037

DnaJ homolog subfamily $C$ member 7

DNAJC7

$6.40 \mathrm{E}-4$

$10 \mathrm{kDa}$ heat shock protein, mitochondrial

HSPE1

0.0016

T-complex protein 1 subunit alpha

TCP1

0.0021

Prefoldin subunit 3

VBP1

$1.45 \mathrm{E}-8$

Glucose metabolism

Hepatocyte nuclear factor 6

ONECUT1

0.0031

Gluconeogenesis and TCA

Malate dehydrogenase cytoplasmic

Malate dehydrogenase mitochondrial

$M D H 1$

$M D H 2$

$1.81 \mathrm{E}-4$

ATP biosynthesis

ATP synthase subunit alpha

ATP5A1 $\quad 0.0011$

ATP synthase subunit gamma

ATP5C1

0.0011

ATP synthase subunit delta

ATP5D

3.59E-5

ATP synthase subunit $f$

ATP5J2

0.0016

ATP synthase subunit $O$

ATP5O

$3.76 \mathrm{E}-5$

NADH dehydrogenase 1 beta subcomplex subunit 10

NDUFB10

$1.59 \mathrm{E}-5$

NADH dehydrogenase 1 beta subcomplex subunit 4

$N A D H$ dehydrogenase 1 beta subcomplex subunit 8

NDUFB4

$1.32 \mathrm{E}-4$

NDUFB8

0.0024

$N A D H$ dehydrogenase [ubiquinone] iron-sulfur protein 3

NDUFS3

$3.59 \mathrm{E}-5$

$\mathrm{NADH}$ dehydrogenase [ubiquinone] iron-sulfur protein 5

NDUFS5

0.0023

Cytochrome b-c1 complex subunit 7

UQCRB

4.05E-5

Table 6: Gene Ontologies over-represented among genes inversely regulated by "hypoxia" and "high dietary lipid" factors

\begin{tabular}{|c|c|c|c|}
\hline System & Gene Ontology & Number of genes & $\begin{array}{c}\text { Corrected } \\
\text { (Benjamini) } \\
\text { p-value } \\
\end{array}$ \\
\hline Cellular component & mitochondrion & 16 & 0.01 \\
\hline Molecular function & $\begin{array}{l}\text { hydrogen ion transporter } \\
\text { activity }\end{array}$ & 8 & 0.01 \\
\hline
\end{tabular}




\section{Figures}

Figure 1: Venn diagram showing the number of genes significantly up and down-regulated by hypoxia and lipid factors.

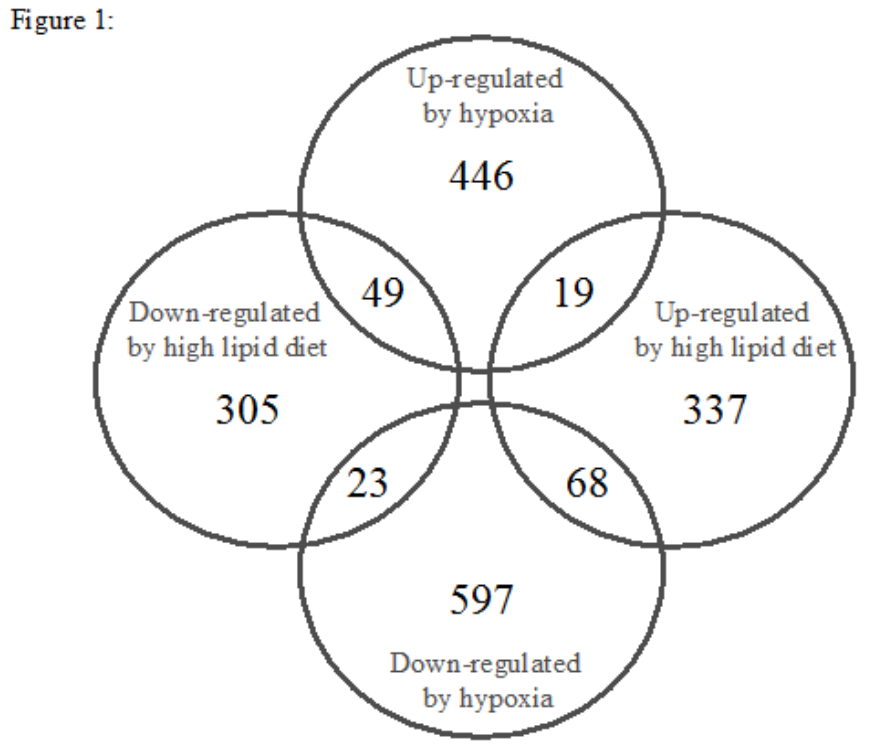


Figure 2: Quantification of threonine 172 phosphorylation in the alpha subunit of AMPK in the Normoxia I-group (NI), Normoxia L-group (NL), Hypoxia l-group $(\mathrm{HI})$ and Hypoxia L-group $(H L)$. Different letters $(a, b)$ indicate a significant difference between the groups $(p<0.05)$.

Figure 2:

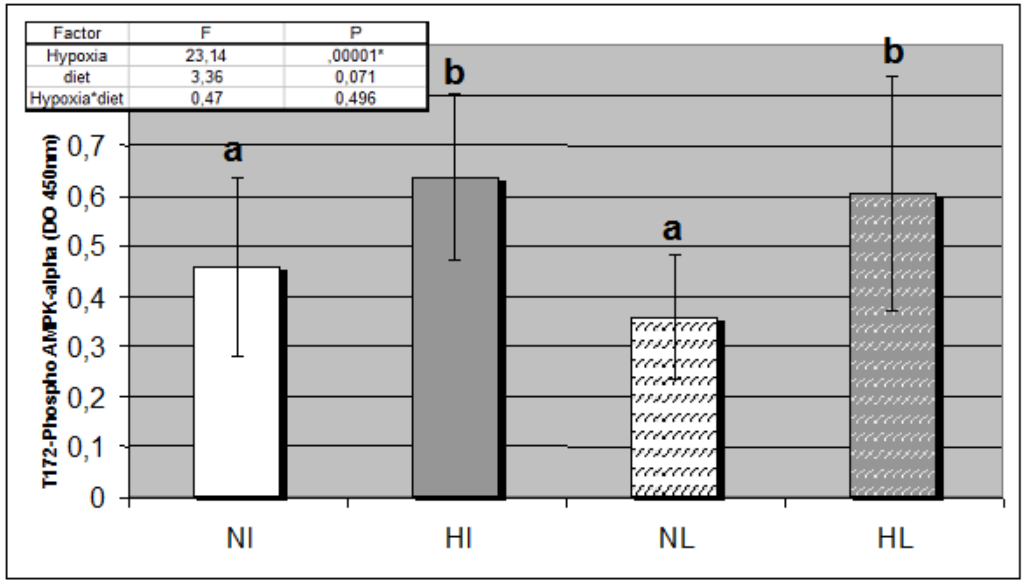

Additional file 1: Western blot analysis from sole tissues performed using Rat Anti-AMPKa

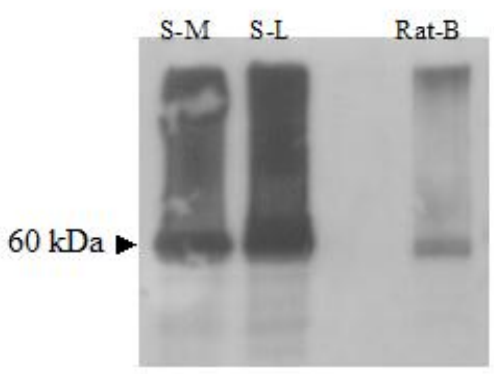

Supplementary data 1: Western blot analysis from protein lysates of sole tissues (sole muscle S-M, sole liver S-L) and rat brain (Rat-B) using Rat AntiAMPK $\alpha$ (23A3) (Rabbit mAb, \#2603, Cell Signaling). A specific band was detected at approximately $60 \mathrm{kDa}$. 
Additional file 2: schematic representation of the approach used for microarray design

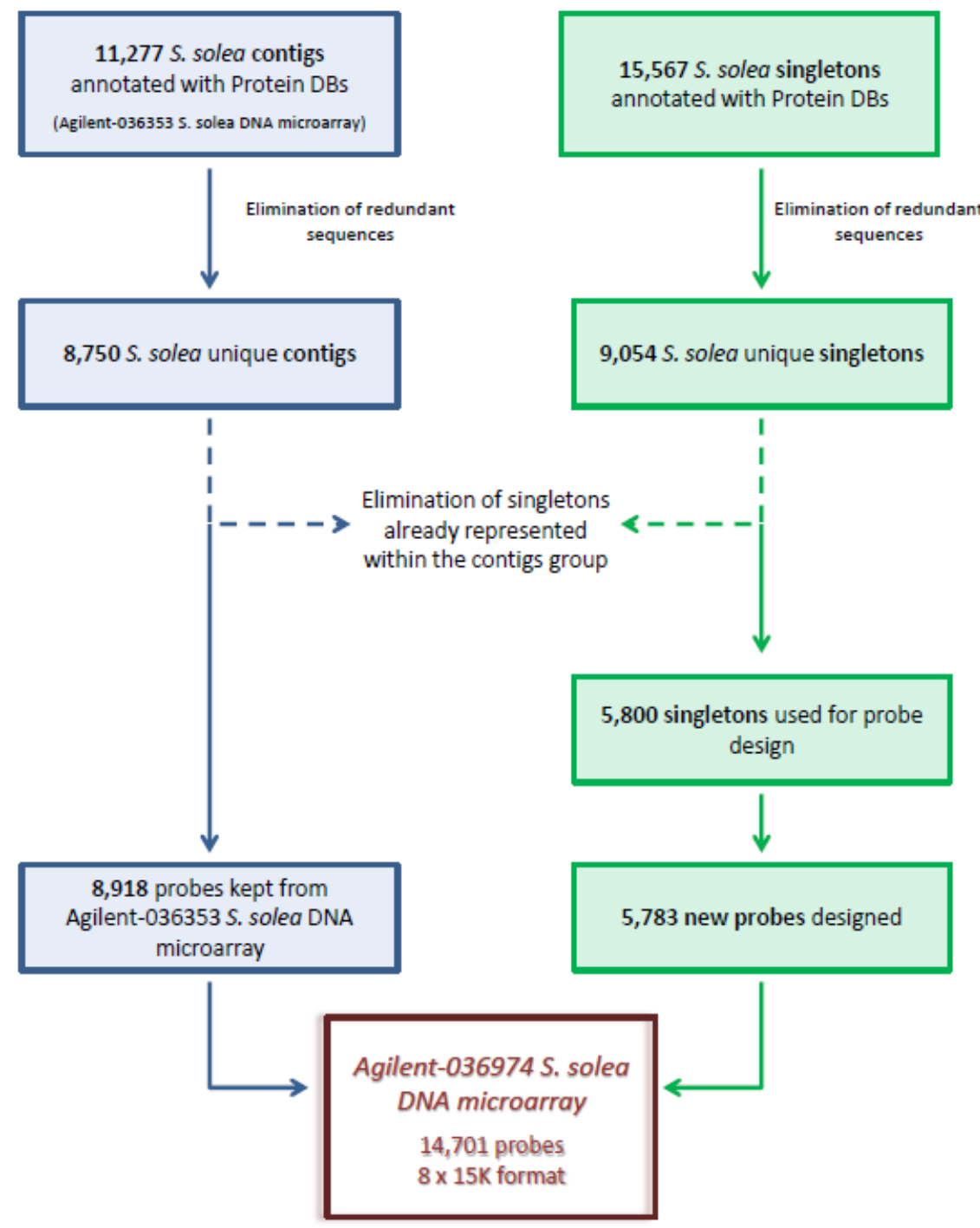

Additional file 3: Full list of genes regulated by "hypoxia", "lipid" and "hypoxia" x "lipid" factors interaction.

List of genes up-regulated by hypoxia $(p<0,005)$

RPS6KA5

SLC44A5

NAA25

TMEM240

CABZ01089777.1

C5H9orf171

K1211

G6W9YEI02J3XJ7

PAPSS1

FGD4

TIA1

SERP1 


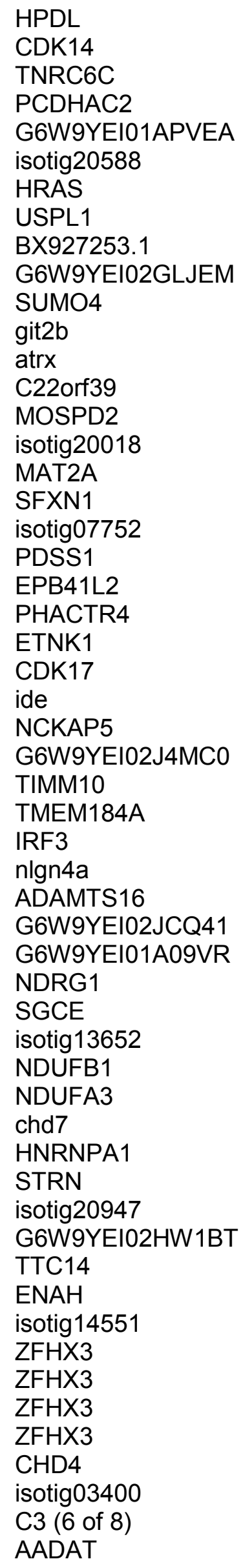


PLRG1

SND1

BX537277.1

DDA1

G6W9YEI01AZVAH

ANKRD11

isotig09984

h2afv

HDAC4

PNP

FGA

PENK

ACVR2B

PDE4DIP

LPL

SLC40A1

slc40a1

G6W9YEI02GCJEH

ASNSD1

DUSP1

POC1B-GALNT4

isotig 13247

ahr2

HIPK3

SERINC5

GADD45G

stat5.1

C1orf116

zmiz1

CSNK1G2

ELMOD2

G6W9YEI02GH9NO

OAT

CTH

F11R

NFIX

plxna4

isotig09491

CU660013.2

SMURF1

ERC1

BX511270.1

BX511270.1

HES5 (7 of 9)

ZNF710

FAM73B

MMP24

CABZ01038928.1

FKBP15

isotig16206

arhgap10

dynll1

ubtfl

SEC16A

G6W9YEI02FM73N 
G6W9YEI01D5RMC

hs3st1l1

FRMD4B

CABZ01118775.1

PLEKHG5

IER5L

LZTR1

S2535

G6W9YEI01BML1Z

G6W9YEI02HXZFW

ALCAM

MAP1LC3C

BX005256.1

PAK2

IRF2BP2

egr1

junbl

FOS

JUNB

isotig05643

isotig03993

ITPKC

fam46c

FAM46A

isotig21478

isotig05160

SLC12A2

MYO9B

FRMPD1

HOXB2

PRKCA

NFE2L2

NFE2L2

SLC43A1

G6W9YEI01CSBV1

hmgb1a

MYCBP2

SLC22A14

PIAS1

RBFOX2

arid1ab

isotig 14780

GLI2

DCN

MYOF

RGS2

isotig20719

syt16

H3F3B

LRP1

RBPMS

FOXF1

ITPK1

SMOC2

Ilgl2 


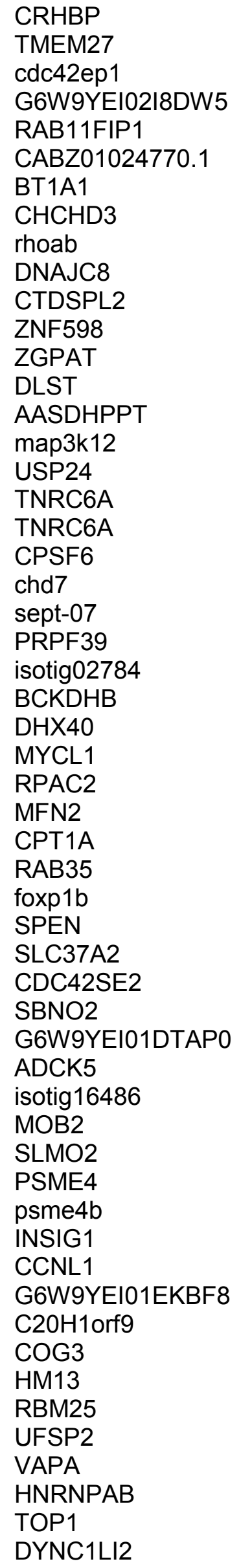


ddx3

isotig18388

$\mathrm{dd} \times 3$

$\mathrm{dd} \times 3$

FBXW11

isotig02477

FARS2

G6W9YEI02GJ4DG

WDR18

ITPR3

BSDC1

RBP5

TULP4

G6W9YEI01A9J45

ABLIM1

pcdh10b

isotig07100

RBM12

MYO7A

AGRN

TRIM29 (7 of 21)

MINK1

PRPSAP1

CTDSPL2

isotig06783

fbxw7

MAN1C1

SLC25A22

isotig06262

epha7

POLB

GYG1

G6W9YEI02H48U0

G6W9YEI01D7VLA

RPS6KA1

TAT

HAL

ucp1

DIABLO

SLC43A1

CBS

CBS

isotig08370

CDO1

AFMID

HACL1

KCNV2

G6W9YEI01A4FFR

atf7ip

nfil3-6

FAM20A

isotig03014

TNRC6C

CSDE1

SDHB 


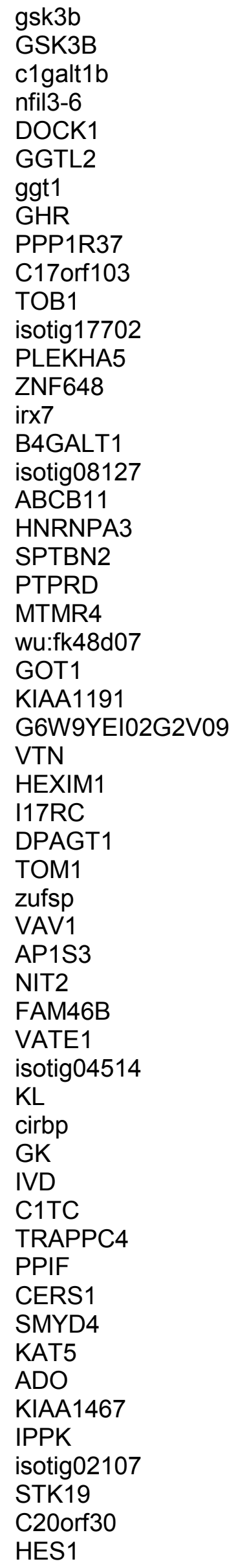


isotig02749

TOPORS

G6W9YEI01EOZR3

IUNH

JAG1

isotig22014

RRM2

ACTL6A

SET

POLE3

isotig12437

HMGB4

APOB (1 of 5)

ACAA2

ACAA2

SRP68

LIN54

CHST15

C1orf50

KIAA1715

G6W9YEI01CV8F2

isotig07264

SLC35A1

TBX1

RFK

C1orf27

BET1

HNRNPAO

DPP3

C5orf43

SGSM3

DERL2

ATOX1

SSR3

ACAD11

isotig08678

isotig12919

HNRNPA1

C16orf58

ARL1

isotig09834

TMEM214

UFSP2

sec22bb

herpud 1

herpud1

G6W9YEI01BYVMH

HSPA5

C20orf24

FICD

SLC35B1

MOGS

LRRC59

SEC61A1

erp44 
PDIA6

G6W9YEI01C0L5P

NEIL3

isotig11784

ERBB4

isotig22007

AL929150.1

GRB10

tdh

slc7a3

SLC7A3

CABZ01044048.1

CABZ01044048.1

PNP

isotig02146

ZFP36

CEBPD

WNK2

G6W9YEI02ITYVR

CREB1

SGK1

keap1a

CR388163.2

G6PC

CYP24A1

G6W9YEI02JCJ2C

ZNF654

HIF3A

FKBP5

DDIT4

dusp6

PCK1

SGK1

FAM160A1

CDH5

RAB14

UBR4

RBMS3

STAB1

ARRB2

gyg2

SYNE1

PEPE

PDGFRB

STARD13

RPL22L1

EEF1B2

RPS10

RS17

RPL28

SLC25A33

IGFBP1

IGFBP1

FAM13B

G6W9YEI01A8WLM 


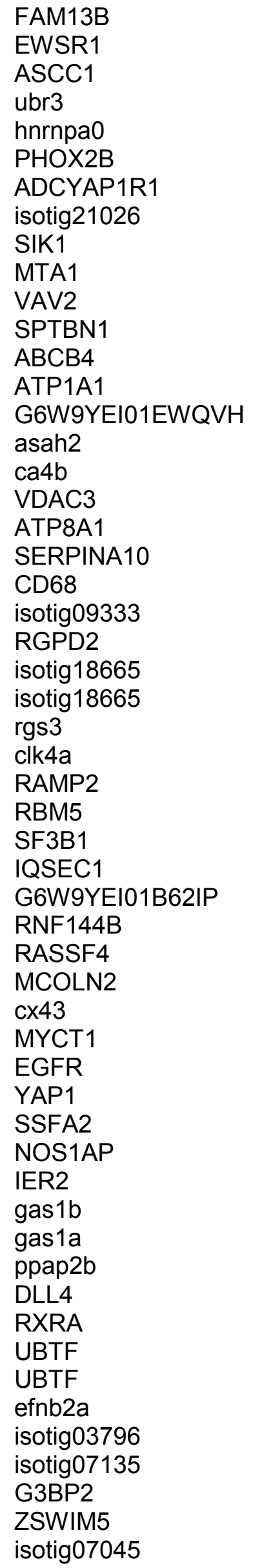


isotig02709

GRB2

ZBED4

btg1

BTG1

PITPNC1

rc3h1

List of genes down-regulated by hypoxia $(p<0,005)$

EPB41

CREBZF

CYP27A1

ubr7

MTHFD2

G6W9YEI02FKBQJ

ELP2

SIRT6

rpp21

agfg1b

ZNF697

AADACL2

isotig03847

DDX42

SENP7

chp2

ANXA3

ANXA3

HBM

TMOD4

isotig21816

rbm38

GAD1

thbs1

F13A1

slc25a37

alox12

ALOX5

jph1a

RHAG

FAM78A

WBP4

GFI1B

isotig18989

SLC4A1

ALAS2

isotig02473

cahz

ba1

ba1

KEL

NDUFV2

BX088712.3

TRA2B

ppp1r10

FHOD3 
LIPS

DOM3Z

PPAP2A

G6W9YEI02J2FXB

SRSF5

SRSF5

LDHB

isotig12370

BX088712.3

LACE1

STRADA

NEK4

METTL5

TM109

CNOT10

CNO

WDR11

CLCN6

isotig07006

isotig06870

CASP2

ZDHHC16

isotig05781

ZNF784

isotig13777

isotig03903

ZNF292

isotig09815

mll4a

GBAS

MEPCE

TASP1

TNPO2

isotig10755

isotig03451

MBD5

BCORL1

Ciz1

PRPF38B

isotig11003

isotig07794

RFX7

GIT2

SRSF9

SLC17A7

$\mathrm{ARIH} 2$

DCTN2

PIP5K1A

TARDBP

RS27

ENOPH1

MAPKAP1

IAH1

GGNBP2

isotig02830 
isotig22031

DOCK1

sox4a

GPR137B

RXRB

ORMDL2

LEPROT

GTF2B

cldn15lb

C21orf2

C21orf2

G6W9YEI02GSPOV

isotig19097

PFKFB4

PKM2

PLOD1

BRD2

TAX1BP1

SLC39A8

isotig00842

TMEM39B

SLC35C1

FBXL20

OTUB2

UPRT

chd2

mxi1

APOA1

apoa4

isotig13249

GSK3A

ncor1

ECE1

$\mathrm{RDH} 14$

KIAA1161

SOD3

DCAF17

TRIM2

FKBP3

NDUFB2

PSMB4

HSPE1

C22orf28

PSMA2

POMP

PSMB5

ap2m1a

ABCC2

TSTD1

GSR

TKTL2

npsn

GSTA

TXNDC2

GSTO2 


\section{ZNF711}

BX470254.2

SNAPC 1

HTD2

CYP8B1

TMC7

BCL6

CXXC5

ncor1

im:7151068

METTL1

TAF10

COX8B

FBXO38

HABP2

G6W9YEI01BR086

TADA3

trpm7

ZMYND8

isotig06993

SRSF11

CCDC115

NDUFS1

HMG20B

CUL4A

UNKL

G6W9YEI01B58UW

NEU1

CK046

ING1

MUTED

CLN5

VPS18

FAM173A

TFDP3

TEX10

H1F0

CPOX

SNX14

CENPM

ero1l

PLCD1

cratb

KDM4C

isotig08263

WDR37

G6W9YEI02FKX89

LDHA

JAKMIP1

KDM5B

eno1

isotig03538

hk2

RSG1

BBS12 


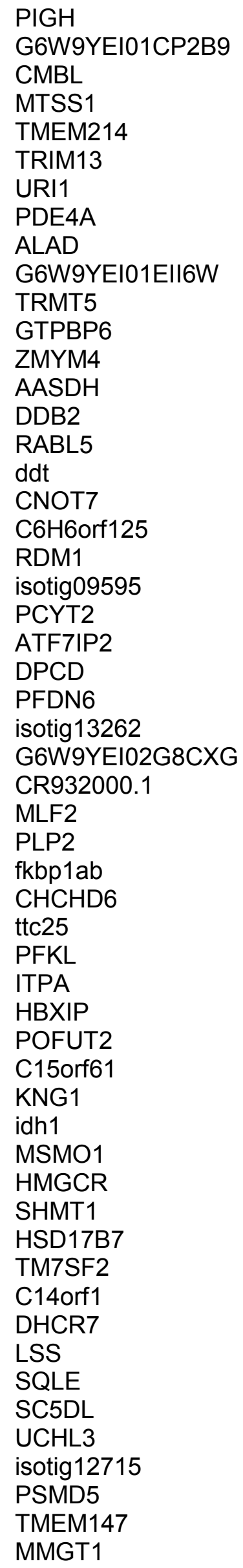


TMEM241

TM111

isotig02419

ZNHIT3

PTPMT1

SRSF7

sf3b14

FDX1L

AATF

TCIRG1

LRG1

isotig06988

selt1a

DCTN1

NAT8

GNPTG

TPC2L

EXOSC7

C18orf21

FHOD1

TPMT

isotig00666

MTX2

rfx2

CHTOP

MRP63

MRPS7

PCGF1

CCBL1

PINX1

isotig00155

UTP6

CCT5

CCT3

$\mathrm{MDH} 2$

VDAC2

ID3

LSM12

CCDC88C

LLPH

RNMTL1

MRPL21

ATP5H

68MP

ATP5J

NDUFB8

ATP5F1

COX5A

ATP5C1

ATP5A1

MDH1

NDUFA10

SLC25A11

METTL20

SLC25A32 


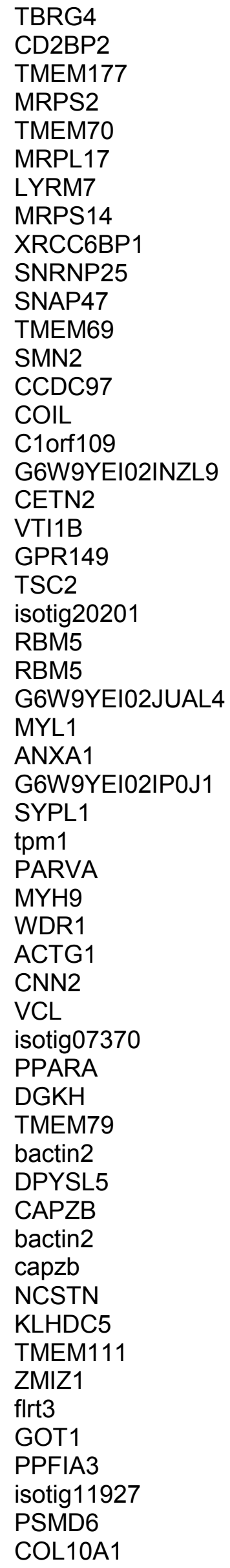


CCDC127

ZHX1-C8ORF76

SRCRB4D

isotig15112

YBEY

FAM102A

TXLNG

RABGAP1L

isotig16608

UCN3

SART1

NAPG

sox6

selt2

sumo3a

PIGV

C19H16orf80

UBE2I

EEF1D

FGF6

ITGA5

LAMB3

FDX1

G6W9YEI02HFJGV

RRN3

WDR19

HDHD3

isotig02411

fabp10a

EIF5

ilf3b

G6W9YEI01CRVFL

MFF

PSME1

AASS

NR2C2AP

VWA1

G6W9YEI02HD500

CECR2

EIF2AK1

PCMT1

METAP2

synj1

TSPAN31

ACTR8

RPAP3

NR2C1

G6W9YEI02182H8

RSAD1

GRHL1

INTS4

BRD9

MED27

TMEM134

IRAK1BP1 


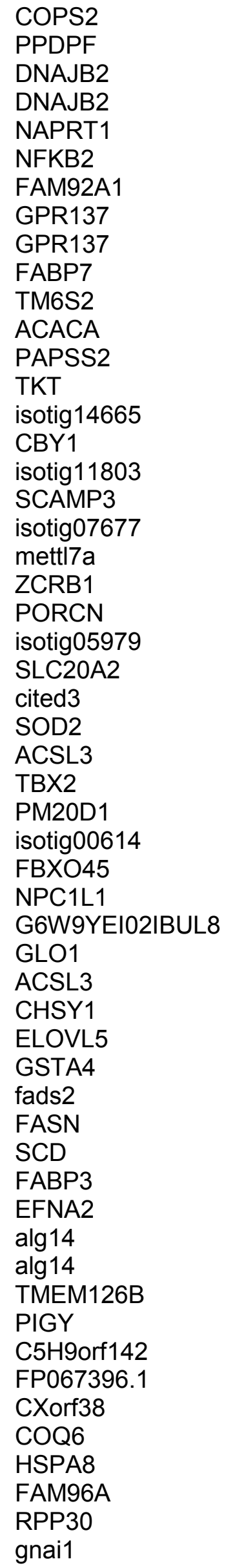


BTBD1

PGRMC1

HNRNPK

C8orf33

MBNL1

CSNK1A1L

tmem106a

GLT1D1

POLR3K

IFRD1

KANK4

MOGAT3

slc35d1a

G6W9YEI02I1LJH

NFKBIL1

UBIAD1

POP7

ENPP5

C9orf46

G6W9YEI01BB8B9

PNPLA3

AGMO

ABHD12B

FRIM

PAIP2B

OAZ1

eno1

$\mathrm{FAH}$

KLF10

MYL7

ICK

TMEM184C

ELOVL6

ACLY

CNP

G6PD

PSME2

CYP2J2 (3 of 6)

CYP2J2 (3 of 6)

CAMKV

cyp3a65

ME1

PGD

G6PD

PRDX3

PQLC3

PSMB7

DKEY-122A22.2

tuba1

tuba1l

RNASEH1

ADCK3

isotig04451

KIAA0913

G6W9YEI01BX5M5 


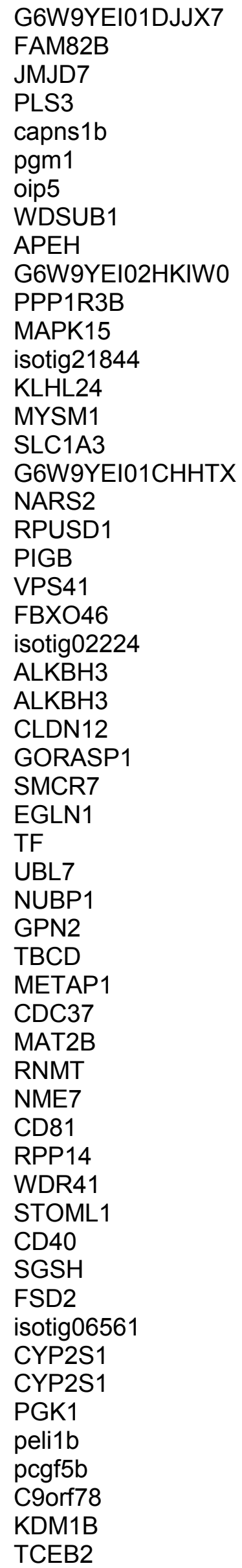




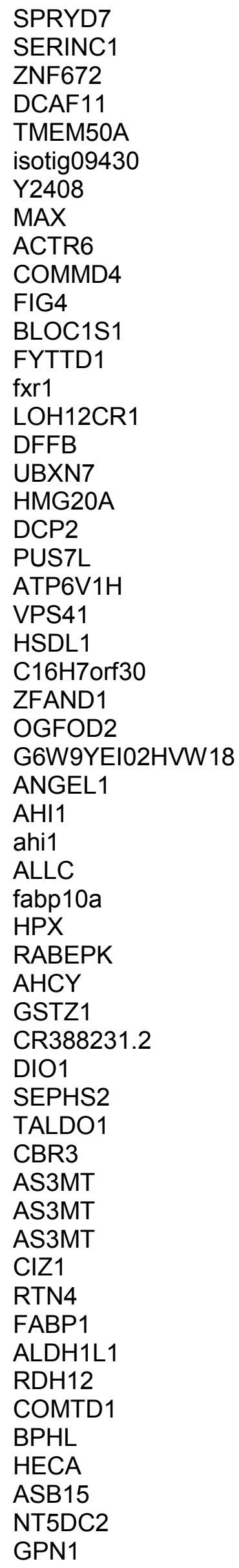


RNF123

gabarapl2

isotig08456

SUMF1

PINK1

TMUB1

BTBD2

C12orf49

FAM168B

BX927314.1

RNF165

RNF165

SSU72

FBXL5

gtf2a1

FBXO18

TDO2

TARDBP

UGDH

WAC

G6W9YEI02FLGRR

isotig 17856

isotig10755

NGDN

G6W9YEI01DKO9B

UBN2

MITD1

BX957329.1

G6W9YEI02IAMBA

CAP2

isotig03223

TMEM9B

ATP6V1H

AMT

CHMP4C

PDCD4

isotig03943

List of genes up-regulated by the high lipid diet $(p<0,005)$

cldni

ATP5G3

G6W9YEI02INLD9

UBTD1

dysfip1

apba2

PVRL1

C3orf17

RLBP1

isotig14534

SNRPE

G6W9YEI02IPW04

RPS27A

SMARCE1

QPRT

ANTXR2 
SQSTM1

DBR1

SCUBE2

luc7|

ITPR1

PLK2

PTPRG

isotig20752

PLEC

isotig20203

RBP5

CHD1

hoxb5a

TTC14

MACD1

RTP3

G6W9YEI02F55KE

HERC5

G6W9YEI01A16BO

ISG15

MIA

SLC25A48

PCDH1

G6W9YEI01DOW7D

atp5ib

C7orf44

QCR10

ugt5c1

PBX4

fgfr2

KIF1B

DNAJC7

isotig08508

POLR1A

PARVA

bactin2

CAP1

G6W9YEI02FLGRR

isotig17856

CNN2

ACTR3B

isotig 10755

UGDH

isotig07006

RBM5

BCORL1

CECR2

WAC

UBR2

GCFC1

ANGPTL4

isotig12954

EPDR1

G6W9YEI01BZAJH

isotig09430 


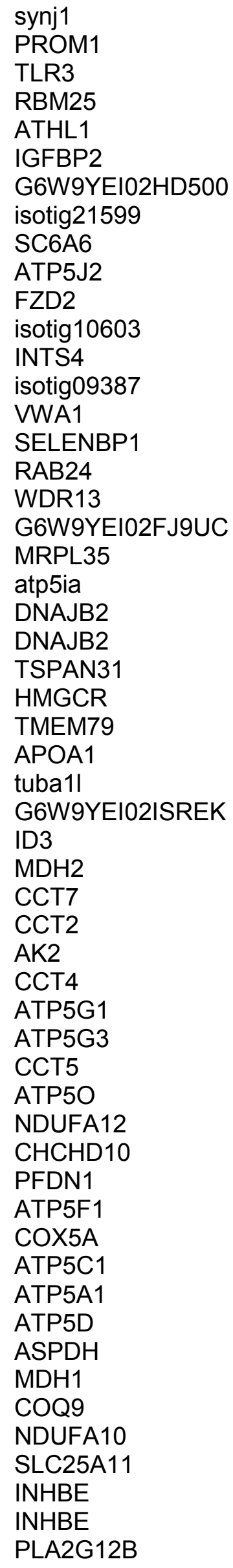


SEC23B

ITSN2

pmt

G6W9YEI02GEBJ2

BLVRA

G6W9YEI02IVFAL

G6W9YEI02JILHM

AGAP9

JUN

TMED5

isotig03795

CCND2

TPD52L2

UQCR 10

ZNF346

SLC1A5

EPN3

SC6A2

BNIP2

isotig 12762

TIMM17A

isotig19865

VPS13B

SERPINE1

HSPA14

TRIM63

isotig07629

SLC6A19

EIF2B5

IPO4

G6W9YEI01C7W27

BAZ1A

FASTK

G6W9YEI0219745

MTMR11

CNOT4

RSF1

C10orf76

NMT1

isotig02038

CABZ01055715.1

WDR60

CC2D2A

HSPE1

TSTD1

C5orf35

isotig16889

MYL7

C7orf25

TXNDC2

AS3MT

AS3MT

TKTL2

npsn

TXNDC2 


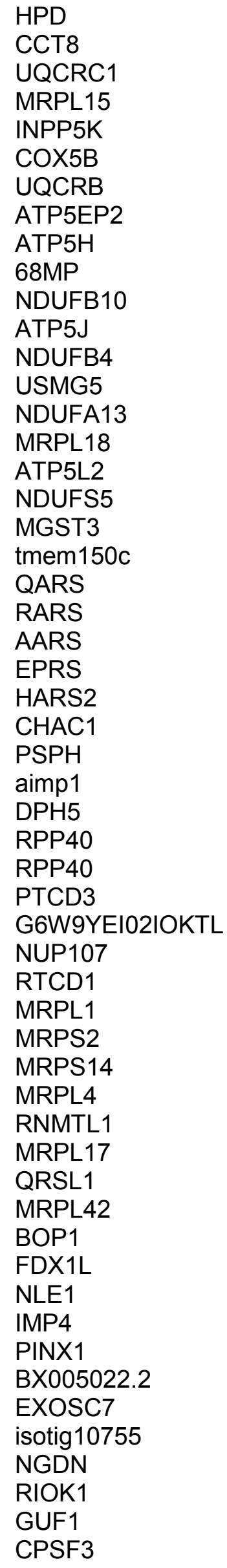


isotig18174

AATF

POLR3F

UTP23

PRKRIP1

ppargc1b

ppargc1b

ppargc1b

SRSF7

MRPS35

WDR74

RG9MTD1

DDX49

NOM1

ATP5J2-PTCD1

BRIX1

MRPS30

RUVBL1

ABT1

CWC15

AHSA1

NIPA1

PDCD11

RBMX2

FBL

WDR36

pprc1

RNF40

ctnnb1

lef1

LEF1

NIPBL

BPTF

MTF2

FITM2

CD68

GET4

SPEN

KIAA2022

DNAJC13

SGPP1

G6W9YEI01ANZJW

ZNF740

PABPN1

FERMT2

FAM161A

LPCAT4

ANGPTL6

MAPT

NT5E

CYP2S1

CYP2S1

DACT2

SLC6A18

CROCC 


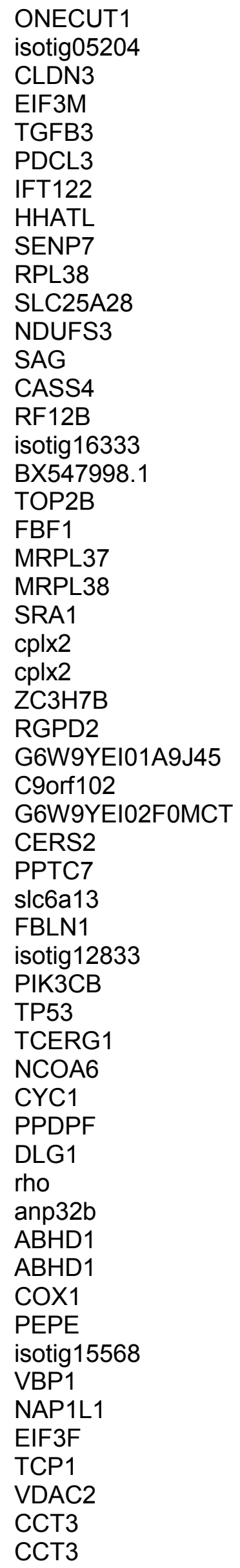


HSP90AB1

eef1g

EIF3H

EIF3K

PNO1

GTPBP4

EIF3A

RPS13

hnrnpa0

mibp2

EIF3B

RPL8

RPL27

eif5a

EEF2

RPL3

pabpc1a

GNB2L1

RPL24

RPL7A

RPL11

RPS15A

RPS3A

RPL35A

rps21

RPS9

RPS2

RPS19

RPL30

rps28

RPL36

RPL32

RPS5

RPL13

RPL18A

RPL7

RPS26

RPL4

IUNH

slc6a13

isotig18625

MAST2

MRPL41

NDUFA6

NDUFB8

SMS

SRSF6

CXorf26

LUZP1

myca

isotig08716

SLIT2

isotig 14230

nsd1a

KIAA0664 


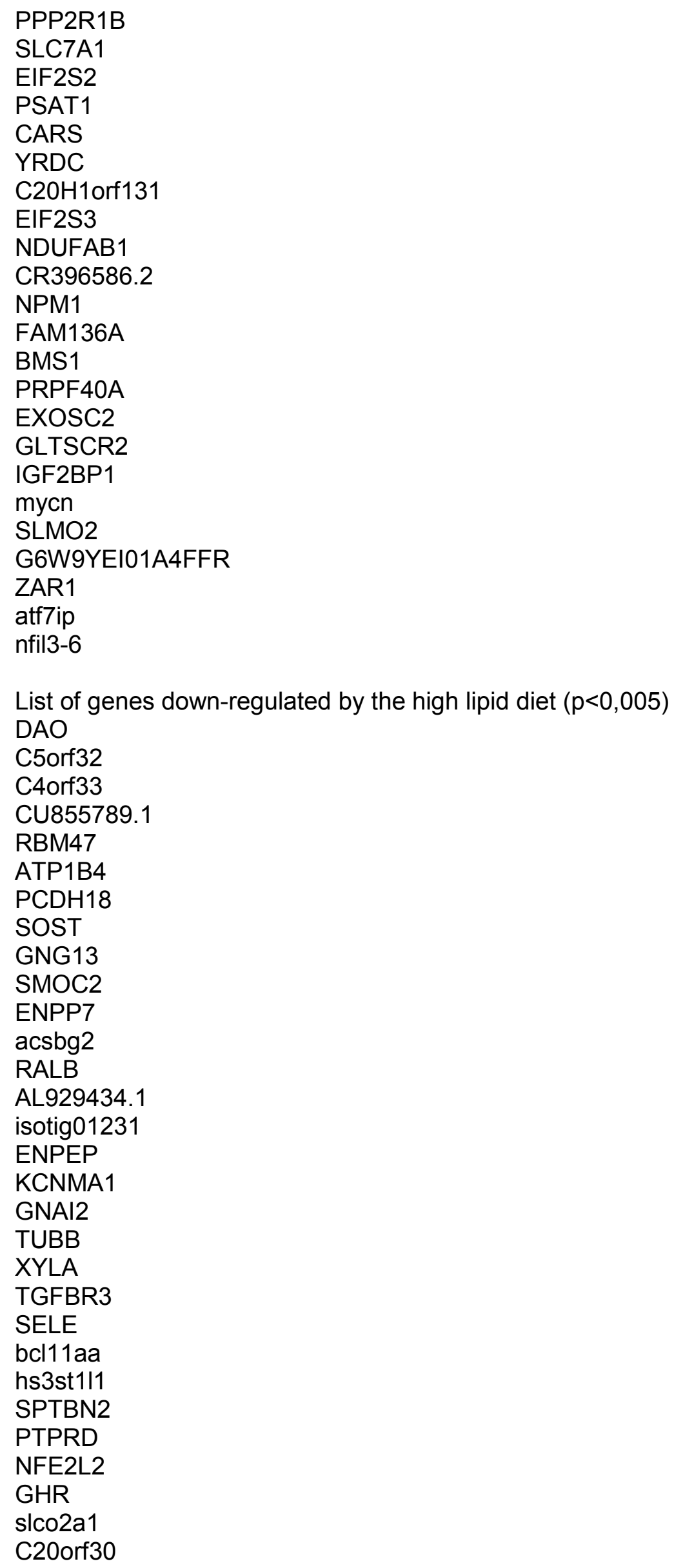


btg1

isotig08370

CDO1

GRB2

PRKCA

gas $1 \mathrm{~b}$

gas $1 a$

ppap2b

efnb2a

zufsp

USP12

isotig13873

DSC1

SGK2

SGK2

isotig03982

khdrbs $1 b$

RNASEH2B

isotig11803

PORCN

DGAT2

RUNX3

nfia

isotig01458

ugt5e1

pik3r1

gpx1b

ahsg

OVGP1 (3 of 5)

OVGP1 (3 of 5)

G6W9YEI02HXFIM

RNF170

GOSR1

FAM96B

IGBP1

VMA21

ETHE1

PTMS

F13A1

MED25

PEX16

BX957234.1

TAF3

ncoa2

CWC25

isotig08966

LOX

ubl3

isotig09571

PRR12

isotig06900

plekhn1

rybpb

IGBP1

NCAPD2 
CR932000.1

$\mathrm{NXPH} 2$

isotig14656

PEX5

isotig05892

sox6

SHD

RAB7A

SMNDC1

KANSL3

UBE2I

HSPB8

$\mathrm{ZC} 4 \mathrm{H} 2$

fkbp1ab

COMTD1

HSBP1

DIDO1

PER2

HNRNPD

AKIP1

USF1

ATP6

CYTB

isotig08422

serpina1

ITIH3

TF

isotig21886

sb:cb37

FGB

FGG

FGA

C8A

CCDC39

selt2

GP1BB

ARHGAP6

GPR89B

G6W9YEI02GWCQG

isotig13920

PPP4R2

EFNA1

FGFR4

isotig21290

PSMD6

PPFIA3

isotig11927

arrdc1b

SYTL2

CLTC

TRPM1

GGT7

PTPN23

HSF1

isotig17631 


\section{OSBPL5}

TECR

Xpo1a

CECR1

PPP2R5E

isotig00711

DNAJC5

G6W9YEI02HTO63

EVL

B3GNTL1

IGF2

PHLPP1

MYO5C

GPM6A

ZNF185

WNT5B

SLC9A6

PCNX

rho

PPP2R2A

PDE4DIP

prdm16

TFCP2L1

TFCP2L1

FMO4

VSTM2A

NFIX

isotig17702

NFIX

isotig14854

TTYH2

TMPRSS4

GALNTL1

cyp1c1

C20orf30

isotig18697

C17orf103

AGXT

SLC2A9

GOT1

foxo3b

GPD1

nucks1a

HSD17B4

HS2ST1

DECR2

HP1BP3

NRARP

nrarpa

C16orf87

SLC22A18

SLC22A18

ALDH7A1

CAMK2D

pglyrp2 
KHK

isotig19287

ZBTB20

F9

ETFA

UCK1

DCTN3

RAD9A

TMCO6

isotig06463

AQP12A

IVD

ANKRD54

POLR2G

LRRC8D

BAP1

isotig06002

isotig04853

G6W9YEI01A7IYK

PDGFRL

AHSG

CYSP1

GMNN

FBX6

BAG2

C11orf54

TMEM59

SDHAF1

G6W9YEI02GW7KA

PEX2

PCBD1

PPP1R37

CUX1

CNPY1

plxna4

GATM

DNAJC9

HDGFL1

$\mathrm{APOH}$

NUDCD2

LYZ

$\mathrm{APOH}$

G6W9YEI02I4IL5

isotig22014

col2a1a

pgm1

RFK

MASP1

FAM53B

nfil3-6

G6W9YEI02GAED7

SEPP1

isotig05773

gna11b

ppp4r2b 
gnb1b

HNF4A

HEXIM1

GPT

CLIC5

SERINC2

ptgds

C5

FTHL17

PPAP2B

DOCK1

GGTL2

ggt1

cfhl2

SERPING1

C1R

IFNAR1

PAM

DAB2

PPP1R3D

PRELID1

ZZEF1

ALD2

TUBB4B

TUBB4A

G6W9YEI01C22WX

AP2S1

G6W9YEI01DGYL6

F9

GGCX

SCAMP2

FAM46A

DNAJC14

isotig16640

YWHAB

BZW1

C5orf43

RNF185

UBA3

CPN2

CPN2

CPN2

SERF2

SNAP29

MCFD2

SRPR

SRPRB

isotig06872

sept-15

isotig11426

OSTC

FKBP14

PARP16

FKBP7

SPCS1 


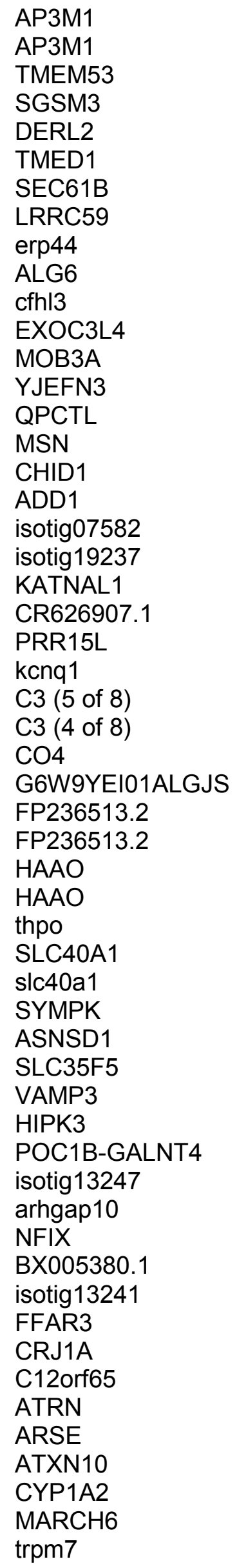




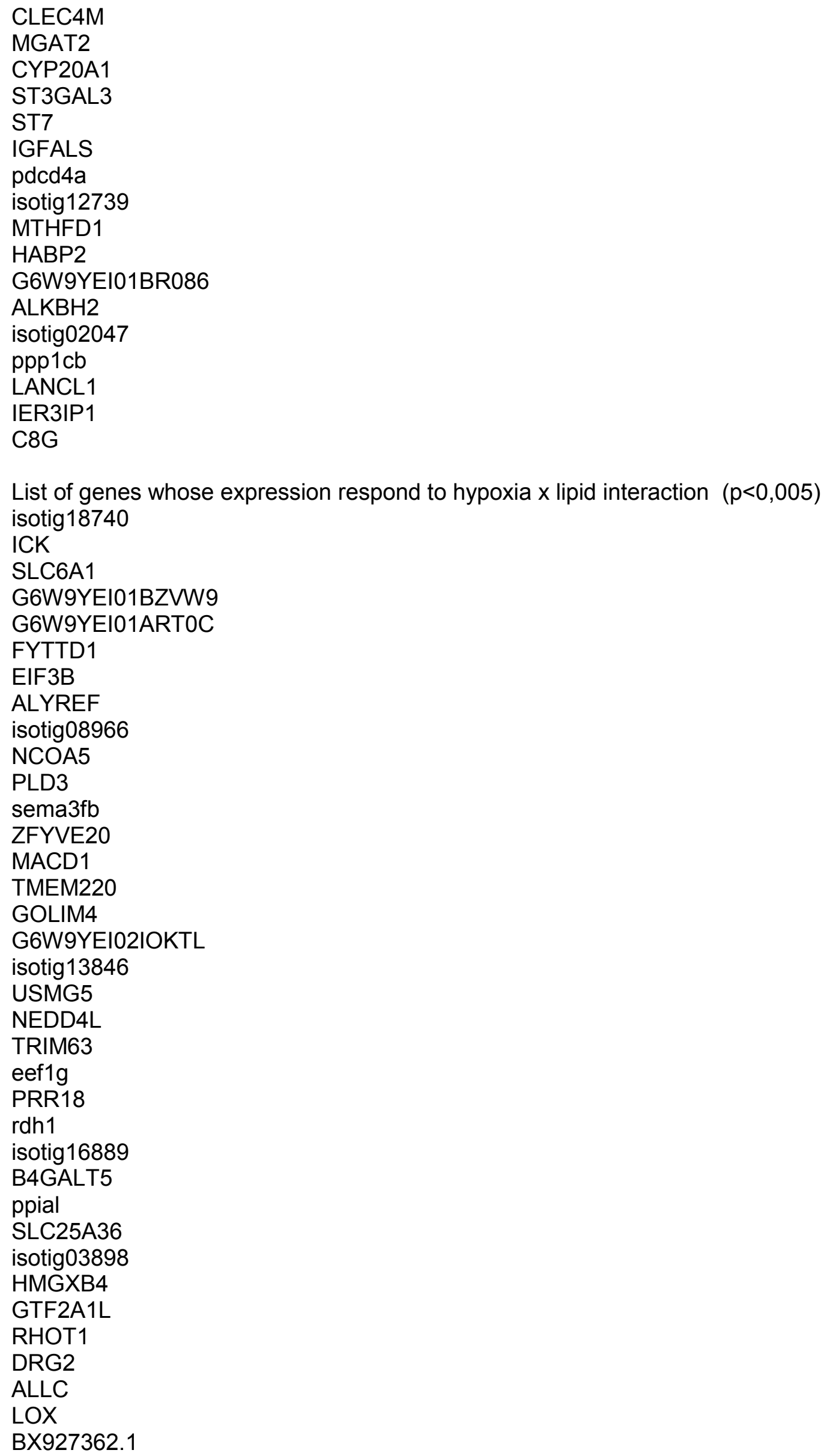

List of genes whose expression respond to hypoxia $x$ lipid interaction $(p<0,005)$ isotig18740

ICK

SLC6A1

G6W9YEI01BZVW9

G6W9YEI01ART0C

FYTTD1

EIF3B

ALYREF

isotig08966

NCOA5

PLD3

sema3fb

ZFYVE20

MACD1

TMEM220

GOLIM4

G6W9YEI02IOKTL

isotig13846

USMG5

NEDD4L

TRIM63

eef1g

PRR18

rdh1

isotig16889

B4GALT5

ppial

SLC25A36

isotig03898

HMGXB4

GTF2A1L

RHOT1

DRG2

ALLC

LOX

BX927362.1 


NOL7
BCL7B
GCA
APTX
TCP11L2
isotig06992
SYF2
DCAF8
TNRC6B
HIBADH
VAMP3
ZRANB1
nPSn
DNM2
TPPP
FRMD8
RANBP3
TCP11
ARHGDIA
C21orf2
XPC
IL16
AIDA
G6W9YEI02F1UZ9
CR392001.1
PDLIM3
FAM195A
G6W9YEI02J3XJ7
TBPL1
G6W9YEI02F219I
C16orf93
VAV1
ARCN1
TMEM214
ELOVL6
CR388231.2
STAT2
OAZ1
C23H20orf24
LCK
GNL3L
POLDIP3
PABPC4
CDC16
MKLN1
LRIG2
GNB2L1
ASB8
ARF4
eno1
WDR77
HNF4A
EPB41
ANGEL1
ANH 10




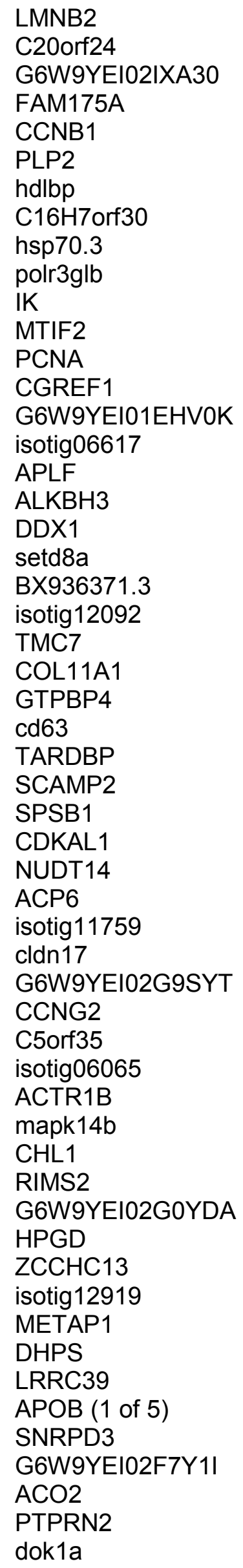




isotig05450
WIPI2
PI4K2B
ASMTL
APLF
G6W9YEI01B58UW
SSRP1
AP1M1
ARL1
isotig18697
CCNDBP1
HSP90AB1
PFN2
NEU1
HEXIM1
ATP2A2
ALKBH3
MYL7
AL929434.1
isotig03341
HDLBP
TXNDC2
TMEM220
PSmd11a
KDELR2
isotig20717
CCND2
UROC1
fancg
TMEM53
hspb11
NPEPL1
C20orf20
RP71-7L19.6
SLC25A39
MAPK15
PDLIM7
fam120c
MKS1
EEF2
KHDRBS1
PEF1
MAPK11
EFTUD2
TMED1
AP2A1
ARR3
pcdh17
PLEC
FAM53B
ISG15
EPB41
GLTSCR2
EIF6


